\title{
EMBEDDING BERGMAN SPACES INTO TENT SPACES
}

\author{
JOSÉ ÁNGEL PELÁEZ, JOUNI RÄTTYÄ, AND KIAN SIERRA
}

\begin{abstract}
Let $A_{\omega}^{p}$ denote the Bergman space in the unit disc $\mathbb{D}$ of the complex plane induced by a radial weight $\omega$ with the doubling property $\int_{r}^{1} \omega(s) d s \leq C \int_{\frac{1+r}{2}}^{1} \omega(s) d s$. The tent space $T_{s}^{q}(\nu, \omega)$ consists of functions such that

$$
\|f\|_{T_{s}^{q}(\nu, \omega)}^{q}=\int_{\mathbb{D}}\left(\int_{\Gamma(\zeta)}|f(z)|^{s} d \nu(z)\right)^{\frac{q}{s}} \omega(\zeta) d A(\zeta)<\infty, \quad 0<q, s<\infty .
$$

Here $\Gamma(\zeta)$ is a non-tangential approach region with vertex $\zeta$ in the punctured unit disc $\mathbb{D} \backslash\{0\}$. We characterize the positive Borel measures $\nu$ such that $A_{\omega}^{p}$ is embedded into the tent space $T_{s}^{q}(\nu, \omega)$, where $1+\frac{s}{p}-\frac{s}{q}>0$, by considering a generalized area operator. The results are provided in terms of Carleson measures for $A_{\omega}^{p}$
\end{abstract}

\section{INTRODUCTION AND MAIN RESULTS}

The theory of tent spaces introduced by Coifman, Meyer and Stein [4, and further studied by Cohn and Verbitsky [3] among others, shows the importance of maximal and square area functions and other objects from harmonic analysis [6] in the study of Hardy spaces in the unit disc $\mathbb{D}=\{z:|z|<1\}$ [5, 7]. The recent studies [12, 13] show that tent spaces have natural analogues for Bergman spaces, and they may play a role in the theory of weighted Bergman spaces similar to that of the original tent spaces in the Hardy space case. The tent space $T_{s}^{q}(\nu, \omega)$ consists of $\nu$-equivalence classes of $\nu$-measurable functions $f: \mathbb{D} \rightarrow \mathbb{C}$ such that

$$
\|f\|_{T_{s}^{q}(\nu, \omega)}^{q}=\left\|A_{s, \nu}(f)\right\|_{L_{\omega}^{q}}^{q}=\int_{\mathbb{D}}\left(\int_{\Gamma(\zeta)}|f(z)|^{s} d \nu(z)\right)^{\frac{q}{s}} \omega(\zeta) d A(\zeta)<\infty, \quad 0<q, s<\infty .
$$

Here $\nu$ is assumed to be a positive Borel measure on $\mathbb{D}$, finite on compact sets, and $\omega \in \widehat{\mathcal{D}}$, that is, $\omega$ is radial and $\widehat{\omega}(r)=\int_{r}^{1} \omega(s) d s$ has the doubling property $\sup _{0 \leq r<1} \widehat{\omega}(r) / \widehat{\omega}\left(\frac{1+r}{2}\right)<\infty$. Moreover,

$$
\Gamma(z)=\left\{\xi \in \mathbb{D}:|\theta-\arg (\xi)|<\frac{1}{2}\left(1-\frac{|\xi|}{r}\right)\right\}, \quad z=r e^{i \theta} \in \overline{\mathbb{D}} \backslash\{0\},
$$

are non-tangential approach regions with vertexes inside the disc, and the related tents are defined by $T(\zeta)=\{z \in \mathbb{D}: \zeta \in \Gamma(z)\}$ for all $\zeta \in \mathbb{D} \backslash\{0\}$. We also set $\omega(T(0))=\lim _{r \rightarrow 0^{+}} \omega(T(r))$ to deal with the origin.

The purpose of this paper is three fold. First, we are interested in the question of when the weighted Bergman space $A_{\omega}^{p}$, consisting of analytic functions in the unit disc $\mathbb{D}$ such that

$$
\|f\|_{A_{\omega}^{p}}^{p}=\int_{\mathbb{D}}|f(z)|^{p} \omega(z) d A(z)<\infty, \quad 0<p<\infty,
$$

Date: July 12, 2018.

Key words and phrases. Tent space, Carleson measure, area operator, integral operator, Bergman space, Hardy space.

This research was supported in part by the Ramón y Cajal program of MICINN (Spain); by Ministerio de Educación y Ciencia, Spain, projects MTM2011-25502 and MTM2011-26538; by La Junta de Andalucía, (FQM210) and (P09-FQM-4468); by Academy of Finland project no. 268009, by Väisälä Foundation of Finnish Academy of Science and Letters, and by Faculty of Science and Forestry of University of Eastern Finland project no. 930349 . 
is continuously or compactly embedded into the tent space $T_{s}^{q}(\nu, \omega)$. Analogous problems for Hardy and Hardy-Sobolev spaces have been considered in [1, 2, 8]. It turns out that the containment $A_{\omega}^{p} \subset T_{s}^{q}(\nu, \omega)$ is naturally described in terms of Carleson measures for $A_{\omega}^{p}$. For $0<p, q<\infty$, a positive Borel measure $\mu$ on $\mathbb{D}$ is a $q$-Carleson measure for $A_{\omega}^{p}$ if there exists a constant $C>0$ such that $\|f\|_{L_{\mu}^{q}} \leq C\|f\|_{A_{\omega}^{p}}$ for all $f \in A_{\omega}^{p}$.

Theorem 1. Let $0<p, q, s<\infty$ such that $1+\frac{s}{p}-\frac{s}{q}>0, \omega \in \widehat{\mathcal{D}}$ and $\nu$ a positive Borel measure on $\mathbb{D}$, finite on compact sets, such that $\nu(\{0\})=0$. Write $\nu_{\omega}(\zeta)=\omega(T(\zeta)) d \nu(\zeta)$ for all $\zeta \in \mathbb{D}$. Then the following assertions hold:

(i) $I_{d}: A_{\omega}^{p} \rightarrow T_{s}^{q}(\nu, \omega)$ is bounded if and only if $\nu_{\omega}$ is a $\left(p+s-\frac{p s}{q}\right)$-Carleson measure for $A_{\omega}^{p}$. Moreover,

$$
\left\|I_{d}\right\|_{A_{\omega}^{p} \rightarrow T_{s}^{q}(\nu, \omega)}^{s} \asymp\left\|I_{d}\right\|_{A_{\omega}^{p} \rightarrow L_{\nu_{\omega}}^{p+s-\frac{p s}{q}}}^{p+s-\frac{p s}{q}} .
$$

(ii) $I_{d}: A_{\omega}^{p} \rightarrow T_{s}^{q}(\nu, \omega)$ is compact if and only if $I_{d}: A_{\omega}^{p} \rightarrow L_{\nu_{\omega}}^{p+s-\frac{p s}{q}}$ is compact.

The requirement $\nu(\{0\})=0$, which does not carry any real restriction, is a technical hypotheses caused by the geometry of the tents $\Gamma(z)$.

Theorem 1 can be interpret as a characterization of Carleson measures. This is the second aim of our study and becomes more apparent when an operator is extracted from Theorem 1 , For $0<s<\infty$, the generalized area operator induced by positive measures $\mu$ and $v$ on $\mathbb{D}$ is defined by

$$
G_{\mu, s}^{v}(f)(z)=\left(\int_{\Gamma(z)}|f(\zeta)|^{s} \frac{d \mu(\zeta)}{v(T(\zeta))}\right)^{\frac{1}{s}}, \quad z \in \mathbb{D} \backslash\{0\} .
$$

Minkowski's inequality shows that $G_{\mu, s}^{v}$ is sublinear if $s \geq 1$. This not the case for $0<s<1$, but instead we have $\left(G_{\mu, s}^{v}(f+g)\right)^{s} \leq\left(G_{\mu, s}^{v}(f)\right)^{s}+\left(G_{\mu, s}^{v}(g)\right)^{s}$. Anyway, we say that $G_{\mu, s}^{v}$ : $A_{\omega}^{p} \rightarrow L_{\omega}^{q}$ is bounded if there exists $C>0$ such that $\left\|G_{\mu, s}^{v}(f)\right\|_{L_{\omega}^{q}} \leq C\|f\|_{A_{\omega}^{p}}$ for all $f \in A_{\omega}^{p}$. Write $\mu_{v}^{\omega}$ for the positive measure such that

$$
d \mu_{v}^{\omega}(z)=\frac{\omega(T(z))}{v(T(z))} d \mu(z)
$$

for $\mu$-almost every $z \in \mathbb{D}$. Fubini's theorem shows that

$$
\begin{aligned}
\left\|G_{\mu, s}^{v}(f)\right\|_{L_{\omega}^{s}}^{s} & =\int_{\mathbb{D}}\left(\int_{\Gamma(z)}|f(\zeta)|^{s} \frac{d \mu(\zeta)}{v(T(\zeta))}\right) \omega(z) d A(z) \\
& =\int_{\mathbb{D}}\left(\int_{\Gamma(z)}|f(\zeta)|^{s} \frac{d \mu_{v}^{\omega}(\zeta)}{\omega(T(\zeta))}\right) \omega(z) d A(z) \\
& =\int_{\mathbb{D} \backslash\{0\}}|f(\zeta)|^{s}\left(\frac{1}{\omega(T(\zeta))} \int_{T(\zeta)} \omega(z) d A(z)\right) d \mu_{v}^{\omega}(\zeta) \\
& =\int_{\mathbb{D} \backslash\{0\}}|f(\zeta)|^{s} d \mu_{v}^{\omega}(\zeta)=\|f\|_{L_{\mu_{v}^{\omega}}^{s}}^{s}-|f(0)|^{s} \mu_{v}^{\omega}(\{0\}),
\end{aligned}
$$

and hence $G_{\mu, s}^{v}: A_{\omega}^{p} \rightarrow L_{\omega}^{s}$ is bounded if and only if $\mu_{v}^{\omega}$ is an $s$-Carleson measure for $A_{\omega}^{p}$. For any $s>0$, we say that $G_{\mu, s}^{v}: A_{\omega}^{p} \rightarrow L_{\mu}^{q}$ is compact if for every bounded sequence $\left\{f_{n}\right\}$ in $A_{\omega}^{p}$ there exists a subsequence $\left\{f_{n_{k}}\right\}$ such that $G_{\mu, s}^{v}\left(f_{n_{k}}\right)$ converges in $L^{q}(\mu)$.

The next theorem gives a characterization of Carleson measures for Bergman spaces by using the generalized area operator $G_{\mu, s}^{v}$. Theorem 1 is a special case of this result, see also Theorem 4 in Section 3 ,

Theorem 2. Let $0<p, q, s<\infty$ such that $s>q-p, \omega \in \widehat{\mathcal{D}}$ and let $\mu, v$ be positive Borel measures on $\mathbb{D}$ such that $\mu(\{z \in \mathbb{D}: v(T(z))=0\})=0=\mu(\{0\})$. Then the following assertions hold: 
(i) $\mu_{v}^{\omega}$ is a q-Carleson measure for $A_{\omega}^{p}$ if and only if $G_{\mu, s}^{v}: A_{\omega}^{p} \rightarrow L_{\omega}^{\frac{p s}{p+s-q}}$ is bounded. Moreover,

$$
\left\|G_{\mu, s}^{v}\right\|_{A_{\omega}^{p} \rightarrow L_{\omega}^{\frac{p s}{p+s-q}}}^{s} \asymp\left\|I_{d}\right\|_{A_{\omega}^{p} \rightarrow L_{\mu_{v}^{\omega}}^{q}}^{q} .
$$

(ii) $I_{d}: A_{\omega}^{p} \rightarrow L_{\mu_{v}^{\omega}}^{q}$ is compact if and only if $G_{\mu, s}^{v}: A_{\omega}^{p} \rightarrow L_{\omega}^{\frac{p s}{p+s-q}}$ is compact.

The third motivation of this study comes from the equivalent $A_{\omega}^{p}$-norm involving square functions, given by

$$
\|f\|_{A_{\omega}^{p}}^{p} \asymp \int_{\mathbb{D}}\left(\int_{\Gamma(\zeta)}\left|D^{\alpha} f(z)\right|^{2}\left(1-\left|\frac{z}{\zeta}\right|\right)^{2 \alpha-2} d A(z)\right)^{\frac{p}{2}} \omega(\zeta) d A(\zeta)+\sum_{j=0}^{[\alpha]-1}\left|f^{(j)}(0)\right|^{p} .
$$

Here $0<\alpha, p<\infty, \omega$ is a radial weight, $D^{\alpha} f$ denotes the fractional derivative of order $\alpha$ and $[\alpha]$ is the integer such that $[\alpha] \leq \alpha<[\alpha]+1$ [12, Theorem 4.2]. This comparability shows that the operator

$$
F_{\alpha}(f)(\zeta)=\left(\int_{\Gamma(\zeta)}\left|D^{\alpha} f(z)\right|^{2}\left(1-\left|\frac{z}{\zeta}\right|\right)^{2 \alpha-2} d A(z)\right)^{\frac{1}{2}}, \quad \zeta \in \mathbb{D} \backslash\{0\},
$$

is bounded from $A_{\omega}^{p}$ to $L_{\omega}^{p}$ for each $\alpha>0$. This is no longer true when $\alpha=0$, and therefore the definition of $G_{\mu, s}^{v}$ is also motivated by the study of this limit case. This was the starting point in the study by Cohn on the area operator $G_{\mu}(f)(z)=\int_{\Gamma(z)}|f(\zeta)| \frac{d \mu(\zeta)}{1-|\zeta|}$, defined for $z$ on the boundary $\mathbb{T}$ of $\mathbb{D}$, acting from the Hardy space $H^{p}$ to $L^{p}(\mathbb{T})$ [2, Theorem 1]. The approach by Cohn relies on ideas by John and Nirenberg [7, Theorem 2.1] and CalderonZygmund decompositions. More recently, similar ideas together the classical factorization of Hardy spaces $H^{p}=H^{p_{1}} \cdot H^{p_{2}}, p^{-1}=p_{1}^{-1}+p_{2}^{-1}$, were used in [8] to study the case $G_{\mu}: H^{p} \rightarrow L^{q}(\mathbb{T})$. We do not employ these techniques in the proof of Theorem 2 but instead we use a description of the boundedness of a weighted maximal function of Hörmander-type. To give the precise statement we need to introduce some notation. The Carleson square $S(I)$ based on an interval $I \subset \mathbb{T}$ is the set $S(I)=\left\{r e^{i t} \in \mathbb{D}: e^{i t} \in I, 1-|I| \leq r<1\right\}$, where $|E|$ denotes the Lebesgue measure of $E \subset \mathbb{T}$. We associate to each $a \in \mathbb{D} \backslash\{0\}$ the interval $I_{a}=\left\{e^{i \theta}:\left|\arg \left(a e^{-i \theta}\right)\right| \leq \frac{1-|a|}{2}\right\}$, and denote $S(a)=S\left(I_{a}\right)$. For a positive Borel measure $\mu$ on $\mathbb{D}$ and $\alpha>0$, define the weighted maximal function

$$
M_{\omega, \alpha}(\mu)(z)=\sup _{z \in S(a)} \frac{\mu(S(a))}{(\omega(S(a)))^{\alpha}}, \quad z \in \mathbb{D} .
$$

In the case $\alpha=1$ simply write $M_{\omega}(\mu)$, and if $\mu$ is of the form $\varphi \omega d A$, then $M_{\omega, \alpha}(\mu)$ is the weighted maximal function $M_{\omega, \alpha}(\varphi)$ of $\varphi$. The following result is [13, Theorem 3].

Theorem A. Let $0<p \leq q<\infty$ and $0<\gamma<\infty$ such that $p \gamma>1$. Let $\omega \in \widehat{\mathcal{D}}$ and $\mu$ be a positive Borel measure on $\mathbb{D}$. Then $\left[M_{\omega}\left((\cdot)^{\frac{1}{\gamma}}\right)\right]^{\gamma}: L_{\omega}^{p} \rightarrow L^{q}(\mu)$ is bounded if and only if $M_{\omega, q / p}(\mu) \in L^{\infty}$. Moreover,

$$
\left\|\left[M_{\omega}\left((\cdot)^{\frac{1}{\gamma}}\right)\right]^{\gamma}\right\|_{\left(L_{\omega}^{p}, L^{q}(\mu)\right)}^{q} \asymp\left\|M_{\omega, q / p}(\mu)\right\|_{L^{\infty}} .
$$

Another maximal operator we will face is defined by $N(f)(z)=\sup _{\zeta \in \Gamma(z)}|f(\zeta)|$. It is known that $N: A_{\omega}^{p} \rightarrow L_{\omega}^{p}$ is bounded for each radial weight $\omega$ and $\|N(f)\|_{L_{\omega}^{p}} \asymp\|f\|_{A_{\omega}^{p}}$ by [12, Lemma 4.4].

Theorems 1 and 2 would perhaps not be of much significance if we did not understand the $q$-Carleson measures for $A_{\omega}^{p}$ sufficiently well. In fact, another important ingredient in the proof of our main results is the following refinement of the characterizations of Carleson measures for Bergman spaces given in [13, Theorem 1]. The estimates for the norm of the identity operator $I_{d}: A_{\omega}^{p} \rightarrow L_{\mu}^{q}$ are of special importance for us. 
Theorem 3. Let $0<p, q<\infty, \omega \in \widehat{\mathcal{D}}$ and let $\mu$ be a positive Borel measure on $\mathbb{D}$. Further, let $d h(z)=d A(z) /\left(1-|z|^{2}\right)^{2}$ denote the hyperbolic measure.

(i) If $p \leq q$, then $\mu$ is a q-Carleson measure for $A_{\omega}^{p}$ if and only if $\sup _{a \in \mathbb{D}} \frac{\mu(S(a))}{\omega(S(a))^{\frac{q}{p}}}<\infty$. Moreover,

$$
\left\|I_{d}\right\|_{A_{\omega}^{p} \rightarrow L_{\mu}^{q}}^{q} \asymp \sup _{a \in \mathbb{D}} \frac{\mu(S(a))}{\omega(S(a))^{\frac{q}{p}}} .
$$

(ii) If $p \leq q$, then $I_{d}: A_{\omega}^{p} \rightarrow L_{\mu}^{q}$ is compact if and only if

$$
\lim _{|a| \rightarrow 1^{-}} \frac{\mu(S(a))}{(\omega(S(a)))^{q / p}}=0 .
$$

(iii) If $q<p$, then the following conditions are equivalent:

(a) $I_{d}: A_{\omega}^{p} \rightarrow L_{\mu}^{q}$ is compact;

(b) $I_{d}: A_{\omega}^{p} \rightarrow L_{\mu}^{q}$ is bounded;

(c) The function

$$
B_{\mu}^{\omega}(z)=\int_{\Gamma(z)} \frac{d \mu(\zeta)}{\omega(T(\zeta))}, \quad z \in \mathbb{D} \backslash\{0\},
$$

belongs to $L_{\omega}^{\frac{p}{p-q}}$

(d) For each fixed $r \in(0,1)$, the function

$$
\Phi_{\mu}^{\omega}(z)=\Phi_{\mu, r}^{\omega}(z)=\int_{\Gamma(z)} \frac{\mu(\Delta(\zeta, r))}{\omega(T(\zeta))} d h(\zeta), \quad z \in \mathbb{D} \backslash\{0\},
$$

belongs to $L_{\omega}^{\frac{p}{p-q}}$;

(e) For each sufficiently large $\lambda=\lambda(\omega)>1$, the function

$$
\Psi_{\mu}^{\omega}(z)=\Psi_{\mu, \lambda}^{\omega}(z)=\int_{\mathbb{D}}\left(\frac{1-|\zeta|}{|1-\bar{z} \zeta|}\right)^{\lambda} \frac{d \mu(\zeta)}{\omega(T(\zeta))}, \quad z \in \mathbb{D}
$$

belongs to $L_{\omega}^{\frac{p}{p-q}}$.

Moreover,

$$
\left\|I_{d}\right\|_{A_{\omega}^{p} \rightarrow L_{\mu}^{q}}^{q} \asymp\left\|M_{\omega}(\mu)\right\|_{L_{\omega}^{\frac{p}{p-q}}} \asymp\left\|B_{\mu}^{\omega}\right\|_{L_{\omega}^{\frac{p}{p-q}}}+\mu(\{0\}) \asymp\left\|\Psi_{\mu}^{\omega}\right\|_{L_{\omega}^{\frac{p}{p-q}}} \asymp\left\|\Phi_{\mu}^{\omega}\right\|_{L_{\omega}^{\frac{p}{p-q}}}+\mu(\{0\}) .
$$

An analogue of the above result for Hardy spaces is essentially known. It can be obtained by using [9, Section 7] and [10. Going further, the implications of the techniques used in this paper can be employed to extend the known results on the area operator on Hardy spaces [2, 8] as well as to the study of the integral operator $T_{g}(f)(z)=\int_{0}^{z} f(\zeta) g^{\prime}(\zeta) d \zeta$ acting on Hardy and Bergman spaces. These results are briefly discussed in Section 5 ,

\section{Carleson measures}

In this section we prove Theorem 3 , For this aim we need some preliminary results and definitions. The following lemma provides useful characterizations of weights in $\widehat{\mathcal{D}}$. For a proof, see [11, 14].

Lemma B. Let $\omega$ be a radial weight. Then the following conditions are equivalent:

(i) $\omega \in \widehat{\mathcal{D}}$;

(ii) There exist $C=C(\omega)>0$ and $\beta=\beta(\omega)>0$ such that

$$
\widehat{\omega}(r) \leq C\left(\frac{1-r}{1-t}\right)^{\beta} \widehat{\omega}(t), \quad 0 \leq r \leq t<1 ;
$$


(iii) There exist $C=C(\omega)>0$ and $\gamma=\gamma(\omega)>0$ such that

$$
\int_{0}^{t}\left(\frac{1-t}{1-s}\right)^{\gamma} \omega(s) d s \leq C \widehat{\omega}(t), \quad 0 \leq t<1
$$

(iv) There exists $\lambda=\lambda(\omega) \geq 0$ such that

$$
\int_{\mathbb{D}} \frac{\omega(z)}{|1-\bar{\zeta} z|^{\lambda+1}} d A(z) \asymp \frac{\widehat{\omega}(\zeta)}{\left(1-|\zeta|^{\lambda}\right.}, \quad \zeta \in \mathbb{D} ;
$$

(v) The associated weight

$$
\omega^{\star}(z)=\int_{|z|}^{1} \omega(s) \log \frac{s}{|z|} s d s, \quad z \in \mathbb{D} \backslash\{0\} .
$$

satisfies

$$
\omega(S(z)) \asymp \omega(T(z)) \asymp \omega^{\star}(z), \quad|z| \rightarrow 1^{-} .
$$

If $\omega \in \widehat{\mathcal{D}}$, then Lemma $\mathbb{B}$ shows that for each $a \in \mathbb{D}$ and $\gamma=\gamma(\omega)>0$ large enough, the function

$$
F_{a, p}(z)=\left(\frac{1-|a|^{2}}{1-\bar{a} z}\right)^{\frac{\gamma+1}{p}}, \quad z \in \mathbb{D},
$$

belongs to $A_{\omega}^{p}$ and satisfies $\left\|F_{a, p}\right\|_{A_{\omega}^{p}}^{p} \asymp \omega(S(a))$ and $\left|F_{a, p}(z)\right| \asymp 1$ for all $z \in S(a)$. This family of test functions will be frequently used in the sequel.

Apart from the tent spaces $T_{s}^{q}(\nu, \omega), 0<q, s<\infty$, defined in the introduction, we will need to consider the case $q=\infty$. For $0<s<\infty$, define

$$
C_{s, \nu}^{s}(f)(\zeta)=\sup _{a \in \Gamma(\zeta)} \frac{1}{\omega(T(a))} \int_{T(a)}|f(z)|^{s} \omega(T(z)) d \nu(z) .
$$

A quasi-norm in the tent space $T_{s}^{\infty}(\nu, \omega)$ is defined by $\|f\|_{T_{s}^{\infty}(\nu, \omega)}=\left\|C_{s, \nu}(f)\right\|_{L^{\infty}}$.

The pseudohyperbolic distance from $z$ to $w$ is defined by $\varrho(z, w)=\left|\frac{1-w}{1-\bar{z} w}\right|$, and the pseudohyperbolic disc of center $a \in \mathbb{D}$ and radius $r \in(0,1)$ is denoted by $\Delta(a, r)=\{z: \varrho(a, z)<r\}$. The Euclidean discs are denoted by $D(a, r)=\{z \in \mathbb{C}:|a-z|<r\}$. Recall that $Z=\left\{z_{k}\right\}_{k=0}^{\infty} \subset$ $\mathbb{D}$ is called a separated sequence if it is separated in the pseudohyperbolic metric, it is an $\varepsilon$-net if $\mathbb{D}=\bigcup_{k=0}^{\infty} \Delta\left(z_{k}, \varepsilon\right)$, and finally it is a $\delta$-lattice if it is a $5 \delta$-net and separated with constant $\gamma=\delta / 5$. If we have a discrete measure $\nu=\sum_{k} \delta_{z_{k}}$, where $\left\{z_{k}\right\}$ is a separated sequence, then we write $T_{q}^{p}(\nu, \omega)=T_{q}^{p}\left(\left\{z_{k}\right\}, \omega\right)$.

Recall that $I_{d}: A_{\omega}^{p} \rightarrow L^{q}(\mu)$ is compact if it maps bounded sets of $A_{\omega}^{p}$ to relatively compact (precompact) sets of $L^{q}(\mu)$. Equivalently, $I_{d}: A_{\omega}^{p} \rightarrow L^{q}(\mu)$ is compact if and only if for every bounded sequence $\left\{f_{n}\right\}$ in $A_{\omega}^{p}$ there exists a subsequence that converges in $L^{q}(\mu)$.

Proof of Theorem 3. (i). There are several ways to bound the operator norm of $I_{d}: A_{\omega}^{p} \rightarrow L_{\mu}^{q}$ from above by the claimed supremum. See [13, Theorem 9] or [11, Theorem 3.3], and also [13, (11)] for the particular case $q=p$. The lower bound is obtained by using test functions, for details, see either [13, Lemma 8] or [11, Theorem 3.3].

(ii). This case can be done by following the proof of [12, Theorem 2.1(ii)], with Lemma B in hand.

(iii). We first show that

$$
\left\|I_{d}\right\|_{A_{\omega}^{p} \rightarrow L_{\mu}^{q}}^{q} \leq\left\|B_{\mu}^{\omega}\right\|_{L_{\omega}^{\frac{p}{p-q}}}+\mu(\{0\}) \asymp\left\|\Psi_{\mu}^{\omega}\right\|_{L_{\omega}^{\frac{p}{p-q}}} \asymp\left\|\Phi_{\mu}^{\omega}\right\|_{L_{\omega}^{\frac{p}{p-q}}}+\mu(\{0\}) \lesssim\left\|M_{\omega}(\mu)\right\|_{L_{\omega}^{\frac{p}{p-q}}} \lesssim\left\|I_{d}\right\|_{A_{\omega}^{p} \rightarrow L_{\mu}^{q}}^{q} .
$$


Fubini's theorem, Hölder's inequality and [12, Lemma 4.4] yield

$$
\begin{aligned}
\|f\|_{L_{\mu}^{q}}^{q} & =\int_{\mathbb{D}}\left(\int_{\Gamma(z)}|f(\zeta)|^{q} \frac{d \mu(\zeta)}{\omega(T(\zeta))}\right) \omega(z) d A(z)+\mu(\{0\})|f(0)|^{q} \\
& \leq \int_{\mathbb{D}} N(f)^{q}(z) \int_{\Gamma(z)} \frac{d \mu(\zeta)}{\omega(T(\zeta))} \omega(z) d A(z)+\mu(\{0\})|f(0)|^{q} \\
& \lesssim\|N(f)\|_{L_{\omega}^{p}}^{q}\left\|B_{\mu}^{\omega}\right\|_{L_{\omega}^{\frac{p}{p-q}}}+\mu(\{0\})\|f\|_{A_{\omega}^{p}}^{q} \asymp\|f\|_{A_{\omega}^{p}}^{q}\left(\left\|B_{\mu}^{\omega}\right\|_{L_{\omega}^{\frac{p}{p-q}}}+\mu(\{0\}),\right.
\end{aligned}
$$

and hence $\left\|I_{d}\right\|_{A_{\omega}^{p} \rightarrow L_{\mu}^{q}}^{q} \lesssim\left\|B_{\mu}^{\omega}\right\|_{L_{\omega}^{\frac{p}{p-q}}}+\mu(\{0\})$. Moreover, $\left\|B_{\mu}^{\omega}\right\|_{L_{\omega}^{\frac{p}{p-q}}}+\mu(\{0\}) \asymp\left\|\Psi_{\mu}^{\omega}\right\|_{L_{\omega}^{\frac{p}{p-q}}}$ by [13, Lemma 4].

Now write $d \nu(z)=\frac{d \mu(z)}{\omega(T(z))}$ and $h_{\lambda}(\zeta, z)=\left(\frac{1-|\zeta|}{|1-\bar{\zeta} z|}\right)^{\lambda}$ for short. Then Fubini's theorem, [13, Lemma 4] and the fact $\omega(T(\zeta)) \asymp \omega(T(u))$ and $h_{\lambda}(\zeta, z) \asymp h_{\lambda}(u, z)$ for $\zeta \in \Delta(u, r), r \in(0,1)$, yield

$$
\begin{aligned}
\left\|\Psi_{\mu}^{\omega}\right\|_{L_{\omega}^{\frac{p}{p-q}}}^{\frac{p}{p-q}} & =\int_{\mathbb{D}}\left(\int_{\mathbb{D}} \frac{h_{\lambda}(\zeta, z)}{|\Delta(\zeta, r)|}|\Delta(\zeta, r)| d \nu(\zeta)\right)^{\frac{p}{p-q}} \omega(z) d A(z) \\
& \asymp \int_{\mathbb{D}}\left(\int_{\mathbb{D}} h_{\lambda}(u, z) \nu(\Delta(u, r)) d h(u)\right)^{\frac{p}{p-q}} \omega(z) d A(z) \\
& \asymp \int_{\mathbb{D}}\left(\int_{\Gamma(z)} \nu(\Delta(u, r)) d h(u)\right)^{\frac{p}{p-q}} \omega(z) d A(z)+\mu(\{0\}) \\
& \asymp \int_{\mathbb{D}}\left(\int_{\Gamma(z)} \frac{\mu(\Delta(u, r))}{\omega(T(u))} d h(u)\right)^{\frac{p}{p-q}} \omega(z) d A(z)+\mu(\{0\}) \\
& =\left\|\Phi_{\mu}^{\omega}\right\|_{L_{\omega}^{\frac{p}{p-q}}}^{\frac{p}{p-q}}+\mu(\{0\})=\|g\|_{T_{1}^{\frac{p}{p-q}}}^{\frac{p}{p-q}(h, \omega)}+\mu(\{0\}),
\end{aligned}
$$

where $g(u)=\frac{\mu(\Delta(u, r))}{\omega(T(u))}$. Now [13, Lemma 7] implies

$$
\|g\|_{T_{1}^{\frac{p}{p-q}}(h, \omega)} \asymp\left\|C_{1, h}(g)\right\|_{L_{\omega}^{\frac{p}{p-q}}} .
$$

Fubini's theorem gives

$$
\begin{aligned}
C_{1, h}(g)(\zeta) & =\sup _{a \in \Gamma(\zeta)} \frac{1}{\omega(T(a))} \int_{T(a)} \mu(\Delta(z, r)) d h(z) \\
& =\sup _{\zeta \in T(a)} \frac{1}{\omega(T(a))} \int_{\mathbb{D}}\left(\int_{T(a) \cap \Delta(u, r)} d h(z)\right) d \mu(u) .
\end{aligned}
$$

The points $u \in \mathbb{D}$ for which $T(a) \cap \Delta(u, r) \neq \emptyset$ are contained in some tent $T\left(a^{\prime}\right)$, where $\arg a^{\prime}=\arg a$ and $1-\left|a^{\prime}\right| \asymp 1-|a|$, for all $a \in \mathbb{D} \backslash D(0, \rho)$, where $\rho=\rho(r) \in(0,1)$. Therefore

$$
\begin{aligned}
\frac{1}{\omega(T(a))} \int_{\mathbb{D}}\left(\int_{T(a) \cap \Delta(u, r)} d h(z)\right) d \mu(u) & =\frac{1}{\omega(T(a))} \int_{T\left(a^{\prime}\right)}\left(\int_{T(a) \cap \Delta(u, r)} d h(z)\right) d \mu(u) \\
& \lesssim \frac{\mu\left(T\left(a^{\prime}\right)\right)}{\omega(T(a))} \asymp \frac{\mu\left(T\left(a^{\prime}\right)\right)}{\omega\left(T\left(a^{\prime}\right)\right)}, \quad a \in \mathbb{D} \backslash D(0, \rho),
\end{aligned}
$$

and it follows that

$$
C_{1, h}(g)(\zeta) \asymp \sup _{\zeta \in T(a)} \frac{\mu(T(a))}{\omega(T(a))} \lesssim M_{\omega}(\mu)(\zeta), \quad \zeta \in \mathbb{D} \backslash\{0\} .
$$


By combining (2.3), (2.4) and (2.5), we deduce

$$
\begin{aligned}
\left\|\Psi_{\mu}^{\omega}\right\|_{L_{\omega}^{\frac{p}{p-q}}} & \asymp\left\|\Phi_{\mu}^{\omega}\right\|_{L_{\omega}^{\frac{p}{p-q}}}+\mu(\{0\})=\|g\|_{T_{1}^{\frac{p}{p-q}}(h, \omega)}+\mu(\{0\}) \\
& \asymp\left\|C_{1, h}(g)\right\|_{L_{\omega}^{\frac{p}{p-q}}}+\mu(\{0\}) \lesssim\left\|M_{\omega}(\mu)\right\|_{L_{\omega}^{\frac{p}{p-q}}} .
\end{aligned}
$$

It remains to prove $\left\|M_{\omega}(\mu)\right\|_{L_{\omega}^{\frac{p}{p-q}}} \lesssim\left\|I_{d}\right\|_{A_{\omega}^{p} \rightarrow L_{\mu}^{q}}^{q}$. To do this, we will show that $M_{\omega}(\mu)$ is pointwise equivalent to the sum of two dyadic maximal functions.

Let $I_{n, k+l}=\left\{e^{i \theta}: \frac{\pi(k+l)}{2^{n+2}} \leq \theta<\frac{\pi(k+l+1)}{2^{n+2}}\right\}$, and denote $\Upsilon_{l}=\left\{I_{n, k+l}: n \in \mathbb{N} \cup\{0\}\right.$, $\left.k=0,1, \ldots, 2^{n+2}-1\right\}$ and $l \in\left\{0, \frac{1}{2}\right\}$. Define the dyadic maximal functions

$$
\widetilde{M}_{\omega, l}^{d}(\mu)(z)=\max \left\{\sup _{z \in T(I), I \in \Upsilon_{l}} \frac{\mu(T(I))}{\omega(T(I))}, \frac{\mu(\mathbb{D})}{\omega(\mathbb{D})}\right\}, \quad z \in \mathbb{D}, \quad l \in\left\{0, \frac{1}{2}\right\},
$$

and set

$$
\widetilde{M}_{\omega}^{d}(\mu)(z)=\widetilde{M}_{\omega, 0}^{d}(\mu)(z)+\widetilde{M}_{\omega, \frac{1}{2}}^{d}(\mu)(z), \quad z \in \mathbb{D} .
$$

If $\omega \in \widehat{\mathcal{D}}$, then $\widetilde{M}_{\omega}^{d}(\mu)(z) \lesssim M_{\omega}(\mu)(z)$ for all $z \in \mathbb{D}$ because $\sup _{I \subset \mathbb{T}} \frac{\omega(S(I))}{\omega(T(I))}<\infty$ by Lemma B For the converse inequality, given $I \subset \mathbb{T}$ such that $z \in S(I)$ there exist intervals $I_{n, k}, I_{n, k+1}$ (if $k=2^{n+2}$, take $I_{n, k}=I_{n, 0}$ ) such that $\left|I_{n+1,0}\right|<|I| \leq\left|I_{n, 0}\right|, I_{n, k} \cap I \neq \emptyset$ and $I_{n, k-1} \cap I=\emptyset$. We may assume that $n \geq 3$, for otherwise the inequality we are searching for is immediate. Then $I \subset I_{n, k} \cup I_{n, k+1}$, and there exists $I_{n-3, m} \in \Upsilon_{0} \cup \Upsilon_{\frac{1}{2}}$ such that $\bigcup_{i=k-2}^{k+3} I_{n, i} \subset I_{n-3, m}$ and $S(I) \subset T\left(I_{n-3, m}\right)$. Therefore Lemma B yields

$$
\frac{\mu(S(I))}{\omega(S(I))} \leq \frac{\mu\left(T\left(I_{n-3, m}\right)\right)}{\omega(T(I))} \leq \frac{\mu\left(T\left(I_{n-3, m}\right)\right)}{\omega\left(T\left(I_{n+1,0}\right)\right)} \asymp \frac{\mu\left(T\left(I_{n-3, m}\right)\right)}{\omega\left(T\left(I_{n-3, m}\right)\right)} \leq \widetilde{M}_{\omega}^{d}(\mu)(z), \quad z \in S(I) .
$$

It follows that $M_{\omega}(\mu)(z) \lesssim \widetilde{M}_{\omega}^{d}(\mu)(z)$, for all $z \in \mathbb{D}$, and hence

$$
M_{\omega}(\mu)(z) \asymp \widetilde{M}_{\omega}^{d}(\mu)(z), \quad z \in \mathbb{D} .
$$

To estimate the norm of $\widetilde{M}_{\omega}^{d}(\mu)$ upwards, let choose $\left\{z_{k}\right\}$ be a separated sequence and define

$$
S_{\lambda}(f)(z)=\sum_{k} f\left(z_{k}\right)\left(\frac{1-\left|z_{k}\right|}{1-\bar{z}_{k} z}\right)^{\lambda}, \quad z \in \mathbb{D} .
$$

By [13, Lemma 6] there exists $\lambda=\lambda(\omega)>1$ such that $S_{\lambda}: T_{2}^{p}\left(\left\{z_{k}\right\}, \omega\right) \rightarrow A_{\omega}^{p}$ is bounded. By denoting $\left\{b_{k}\right\}=\left\{f\left(z_{k}\right)\right\}$, this implies

$$
\int_{\mathbb{D}}\left|\sum_{k} b_{k} h_{\lambda}\left(z_{k}, z\right)\right|^{q} d \mu(z)=\left\|S_{\lambda}(f)\right\|_{L^{q}(\mu)}^{q} \lesssim\left\|I_{d}\right\|_{A_{\omega}^{p} \rightarrow L_{\mu}^{q}}^{q}\left\|S_{\lambda}\right\|_{T_{2}^{p}\left(\left\{z_{k}\right\}, \omega\right) \rightarrow A_{\omega}^{p}}^{q}\left\|\left\{b_{k}\right\}\right\|_{T_{2}^{p}\left(\left\{z_{k}\right\}, \omega\right)}^{q} .
$$

By replacing $b_{k}$ by $r_{k}(t) b_{k}$, where $r_{k}$ denotes the $k$ th Rademacher function, using the fact that $\left|h_{\lambda}\left(z_{k}, z\right)\right| \gtrsim \chi_{T\left(z_{k}\right)}(z)$ for $z \in T\left(z_{k}\right)$, and applying Khinchine's inequality, we deduce

$$
\int_{\mathbb{D}}\left(\sum_{k}\left|b_{k}\right|^{2} \chi_{T\left(z_{k}\right)}(z)\right)^{\frac{q}{2}} d \mu(z) \lesssim\left\|I_{d}\right\|_{A_{\omega}^{p} \rightarrow L_{\mu}^{q}}^{q}\left\|S_{\lambda}\right\|_{T_{2}^{p}\left(\left\{z_{k}\right\}, \omega\right) \rightarrow A_{\omega}^{p}}^{q}\left\|\left\{b_{k}\right\}\right\|_{T_{2}^{p}\left(\left\{z_{k}\right\}, \omega\right)}^{q} .
$$

Let $l \in\left\{0, \frac{1}{2}\right\}$ be fixed. For each $k \in \mathbb{Z}$, let $\mathcal{E}_{k}$ denote the collection of maximal dyadic tents $T \in\left\{T(I): I \in \Upsilon_{l}\right\} \cup\{\mathbb{D}\}$ with respect to inclusion such that $\mu(T)>2^{k} \omega(T)$, and let $E_{k}=\cup_{T \in \mathcal{E}_{k}} T$. Then $2^{k}<\widetilde{M}_{\omega, l}^{d}(\mu)(z) \leq 2^{k+1}$ for $z \in E_{k} \backslash E_{k+1}$. Let now $\left\{b_{T}\right\}$ be a sequence indexed by $T \in \mathcal{E}=\cup_{k} \mathcal{E}_{k}$. Assume for a moment that $\mu$ has compact support. Then $\left\{b_{T}\right\}$ is a finite sequence. For $T \in \mathcal{E}$, let $G(T)=T-\cup\left\{T^{\prime} \in \mathcal{E}: T^{\prime} \subsetneq T\right\}$, and hence 
$G(T)=T-\cup\left\{T^{\prime} \in \mathcal{E}_{k+1}: T^{\prime} \subsetneq T\right\}$ for $T \in \mathcal{E}_{k}$. If $T_{1}, T_{2} \in \mathcal{E}$ are different (either one is strictly included in the other or they are disjoint), the sets $G\left(T_{1}\right)$ and $G\left(T_{2}\right)$ are disjoint, and hence

$$
\left(\sum_{T \in \mathcal{E}}\left|b_{T}\right|^{2} \chi_{T}(z)\right)^{\frac{q}{2}} \geq\left(\sum_{T \in \mathcal{E}}\left|b_{T}\right|^{2} \chi_{G(T)}(z)\right)^{\frac{q}{2}}=\sum_{T \in \mathcal{E}}\left|b_{T}\right|^{q} \chi_{G(T)}(z) .
$$

Index the tents in $\mathcal{E}$ according to which $\mathcal{E}_{k}$ with maximal index they belong to, by writing $\mathcal{E}_{k} \backslash \cup_{m<k} \mathcal{E}_{m}=\left\{T_{j}^{k}: j \in \mathbb{N}\right\}$. Further, denote $b_{k, j}=b_{T_{j}^{k}}$ and let $z_{k, j}$ denote the vertex of $T_{j}^{k}$ i.e. $T_{j}^{k}=T\left(z_{k, j}\right)$ (with the convenience that the vertex of $\mathbb{D}$ is the origin). The estimates (2.7) and (2.8) yield

$$
\begin{gathered}
\sum_{k, j} b_{k, j}^{q}\left(\mu\left(T_{j}^{k}\right)-\sum_{T_{i}^{k+1} \subset T_{j}^{k}} \mu\left(T_{i}^{k+1}\right)\right) \lesssim\left\|I_{d}\right\|_{A_{\omega}^{p} \rightarrow L_{\mu}^{q}}^{q}\left\|\left\{b_{k, j}\right\}\right\|_{T_{2}^{p}\left(\left\{z_{k, j}\right\}, \omega\right)}^{q} \\
=\left\|I_{d}\right\|_{A_{\omega}^{p} \rightarrow L_{\mu}^{q}}^{q}\left(\int_{\mathbb{D}}\left(\sum_{k, j}\left|b_{k, j}\right|^{2} \chi_{T_{j}^{k}}(z)\right)^{\frac{p}{2}} \omega(z) d A(z)\right)^{\frac{q}{p}} .
\end{gathered}
$$

Write $r=\frac{p}{q}$ for short, and choose $b_{k, j}^{q}=2^{k\left(r^{\prime}-1\right)}$ for each $k$ and $j$. Then, by using the inequality $2^{k}<\widetilde{M}_{\omega, l}^{d}(\mu)(z) \leq 2^{k+1}$ for $z \in E_{k} \backslash E_{k+1}$, the left hand side of (2.9) can be estimated as

$$
\begin{aligned}
& \sum_{k, j} b_{k, j}^{q}\left(\mu\left(T_{j}^{k}\right)-\sum_{T_{i}^{k+1} \subset T_{j}^{k}} \mu\left(T_{i}^{k+1}\right)\right)=\sum_{k} 2^{k\left(r^{\prime}-1\right)} \mu\left(E_{k}\right)-\sum_{k} 2^{k\left(r^{\prime}-1\right)} \sum_{j} \mu\left(T_{j}^{k} \cap E_{k+1}\right) \\
& =\sum_{k}\left(2^{k\left(r^{\prime}-1\right)}-2^{(k-1)\left(r^{\prime}-1\right)}\right) \mu\left(E_{k}\right)=\left(1-\frac{1}{2^{r^{\prime}-1}}\right) \sum_{k} 2^{k r^{\prime}} 2^{-k} \sum_{j} \frac{\mu\left(T_{j}^{k}\right)}{\omega\left(T_{j}^{k}\right)} \omega\left(T_{j}^{k}\right) \\
& \gtrsim \sum_{k} 2^{k r^{\prime}} \omega\left(E_{k}\right) \gtrsim \sum_{k} \int_{E_{k} \backslash E_{k+1}}\left(\widetilde{M}_{\omega, l}^{d}(\mu)(z)\right)^{r^{\prime}} \omega(z) d A(z)=\left\|\widetilde{M}_{\omega, l}^{d}(\mu)\right\|_{L_{\omega}^{r^{\prime}}}^{r^{\prime}},
\end{aligned}
$$

while the integral on the right hand side of (2.9) with the notation $\eta=2^{\left(r^{\prime}-1\right) \frac{2}{q}}$ becomes

$$
\begin{aligned}
& \int_{\mathbb{D}}\left(\sum_{k, j}\left|b_{k, j}\right|^{2} \chi_{T_{j}^{k}}(z)\right)^{\frac{p}{2}} \omega(z) d A(z)=\int_{\mathbb{D}}\left(\sum_{k, j} \eta^{k} \chi_{T_{j}^{k}}(z)\right)^{\frac{p}{2}} \omega(z) d A(z) \\
& =\int_{\mathbb{D}}\left(\sum_{k} \eta^{k} \chi_{E_{k}}(z)\right)^{\frac{p}{2}} \omega(z) d A(z)=\int_{\mathbb{D}}\left(\frac{\eta}{\eta-1} \sum_{k}\left(\eta^{k}-\eta^{k-1}\right) \chi_{E_{k}}(z)\right)^{\frac{p}{2}} \omega(z) d A(z) \\
& \asymp \int_{\mathbb{D}}\left(\sum_{k} \eta^{k}\left(\chi_{E_{k}}(z)-\chi_{E_{k+1}}(z)\right)\right)^{\frac{p}{2}} \omega(z) d A(z)=\int_{\mathbb{D}}\left(\sum_{k} \eta^{k} \chi_{E_{k} \backslash E_{k+1}}(z)\right)^{\frac{p}{2}} \omega(z) d A(z) \\
& =\int_{\mathbb{D}} \sum_{k} \eta^{\frac{k p}{2}} \chi_{E_{k} \backslash E_{k+1}}(z) \omega(z) d A(z)=\int_{\mathbb{D}} \sum_{k} 2^{r^{\prime} k} \chi_{E_{k} \backslash E_{k+1}}(z) \omega(z) d A(z) \\
& \asymp \sum_{k} \int_{E_{k} \backslash E_{k+1}}\left(\widetilde{M}_{\omega, l}^{d}(\mu)(z)\right)^{r^{\prime}} \omega(z) d A(z)=\left\|\widetilde{M}_{\omega, l}^{d}(\mu)\right\|_{L_{\omega}^{\prime \prime}}^{r^{\prime}}
\end{aligned}
$$

Consequently, $\left\|\widetilde{M}_{\omega, l}^{d}(\mu)\right\|_{L_{\omega}^{r^{\prime}}}^{r^{\prime}} \lesssim\left\|I_{d}\right\|_{A_{\omega}^{p} \rightarrow L_{\mu}^{q}}^{q}\left\|\widetilde{M}_{\omega, l}^{d}(\mu)\right\|_{L_{\omega}^{r^{\prime}}}^{\frac{r^{\prime}}{r}}$ and thus $\left\|\widetilde{M}_{\omega, l}^{d}(\mu)\right\|_{L_{\omega}^{\frac{p}{p-q}}} \lesssim\left\|I_{d}\right\|_{A_{\omega}^{p} \rightarrow L_{\mu}^{q}}^{q}$ because $r^{\prime}=\left(\frac{p}{q}\right)^{\prime}=\frac{p}{p-q}$. Since this is valid for $l \in\left\{0, \frac{1}{2}\right\}$, using Minkowski's inequality and 
(2.6) we get

$$
\left\|M_{\omega}(\mu)\right\|_{L_{\omega}^{\frac{p}{p-q}}} \asymp\left\|\widetilde{M}_{\omega}^{d}(\mu)\right\|_{L_{\omega}^{\frac{p}{p-q}}} \leq\left\|\widetilde{M}_{\omega, 0}^{d}(\mu)\right\|_{L_{\omega}^{\frac{p}{p-q}}}+\left\|\widetilde{M}_{\omega, \frac{1}{2}}^{d}(\mu)\right\|_{L_{\omega}^{\frac{p}{p-q}}} \lesssim\left\|I_{d}\right\|_{A_{\omega}^{p} \rightarrow L_{\mu}^{q}}^{q}
$$

for $\mu$ with compact support. If $\mu$ is positive, then the above estimate, applied to the compactly supported $\mu_{r}=\chi_{D(0, r)} \mu$, and the standard limiting argument with monotone convergence theorem gives $\left\|M_{\omega}(\mu)\right\|_{L_{\omega}^{p-q}} \lesssim\left\|I_{d}\right\|_{A_{\omega}^{p} \rightarrow L_{\mu}^{q}}^{q}$. Hence the claimed operator norm estimates are valid and, in particular, (b)-(e) are equivalent.

To complete the proof of (iii), it suffices to show that $I_{d}: A_{\omega}^{p} \rightarrow L_{\mu}^{q}$ is compact if (e) is satisfied. By the hypothesis (e), (2.3) and the dominated convergence theorem,

$$
\begin{aligned}
0 & =\lim _{R \rightarrow 1^{-}} \int_{\mathbb{D}}\left(\int_{\{R<|z|<1\}}\left(\frac{1-|z|}{|1-\bar{\zeta} z|}\right)^{\lambda} \frac{d \mu(z)}{\omega(T(z))}\right)^{\frac{p}{p-q}} \omega(\zeta) d A(\zeta) \\
& \gtrsim \lim _{R \rightarrow 1^{-}} \int_{\mathbb{D}}\left(\int_{\Gamma(\zeta) \backslash \overline{D(0, R)}} \frac{d \mu(z)}{\omega(T(z))}\right)^{\frac{p}{p-q}} \omega(\zeta) d A(\zeta) .
\end{aligned}
$$

Let $\left\{f_{n}\right\}$ be a bounded sequence in $A_{\omega}^{p}$. Then $\left\{f_{n}\right\}$ is locally bounded and thus constitutes a normal family. Hence we may extract a subsequence $\left\{f_{n_{k}}\right\}$ that converges uniformly on compact subsets of $\mathbb{D}$ to $f \in A_{\omega}^{p}$. Write $g_{k}=f_{n_{k}}-f$. For $\varepsilon>0$, by (2.10), there exists $R_{0}=R_{0} \in(0,1)$ such that

$$
\int_{\mathbb{D}}\left(\int_{\Gamma(\zeta) \backslash \overline{D\left(0, R_{0}\right)}} \frac{d \mu(z)}{\omega(T(z))}\right)^{\frac{p}{p-q}} \omega(\zeta) d A(\zeta)<\varepsilon^{\frac{p}{p-q}}
$$

By the uniform convergence, we may choose $k_{0} \in \mathbb{N}$ such that $\left|g_{k}(z)\right|<\varepsilon^{1 / q}$ for all $k \geq k_{0}$ and $z \in \overline{D\left(0, R_{0}\right)}$. Then Fubini's theorem, Hölder's inequality and [12, Lemma 4.4] yield

$$
\begin{aligned}
& \left\|g_{k}\right\|_{L_{\mu}^{q}}^{q}=\int_{\frac{D\left(0, R_{0}\right)}{}}\left|g_{k}(\zeta)\right|^{q} d \mu(\zeta)+\int_{\mathbb{D} \backslash \overline{D\left(0, R_{0}\right)}}\left|g_{k}(\zeta)\right|^{q} d \mu(\zeta) \\
& \leq \varepsilon \mu(\mathbb{D})+\int_{\mathbb{D}}\left(\int_{\Gamma(z) \backslash \overline{D\left(0, R_{0}\right)}}\left|g_{k}(\zeta)\right|^{q} \frac{d \mu(\zeta)}{\omega(T(\zeta))}\right) \omega(z) d A(z) \\
& \leq \varepsilon \mu(\mathbb{D})+\int_{\mathbb{D}} N\left(g_{k}\right)^{q}(z)\left(\int_{\Gamma(z) \backslash \overline{D\left(0, R_{0}\right)}} \frac{d \mu(\zeta)}{\omega(T(\zeta))}\right) \omega(z) d A(z) \\
& \leq \varepsilon \mu(\mathbb{D})+\left\|N\left(g_{k}\right)\right\|_{L_{\omega}^{p}}^{q} \varepsilon \asymp \varepsilon \mu(\mathbb{D})+\left\|g_{k}\right\|_{A_{\omega}^{p} \varepsilon \lesssim \varepsilon,}^{q}
\end{aligned}
$$

and thus $I_{d}: A_{\omega}^{p} \rightarrow L_{\mu}^{q}$ is compact.

\section{Bounded operators $G_{\mu, s}^{v}: A_{\omega}^{p} \rightarrow L_{\omega}^{q}$}

Theorem 2 is equivalent to the following result.

Theorem 4. Let $0<p, q, s<\infty$ such that $1+\frac{s}{p}-\frac{s}{q}>0, \omega \in \widehat{\mathcal{D}}$ and let $\mu, v$ be positive Borel measures on $\mathbb{D}$ such that $\mu(\{z \in \mathbb{D}: v(T(z))=0\})=0=\mu(\{0\})$. Then the following assertions hold:

(i) $G_{\mu, s}^{v}: A_{\omega}^{p} \rightarrow L_{\omega}^{q}$ is bounded if and only if $\mu_{v}^{\omega}$ is a $\left(p+s-\frac{p s}{q}\right)$-Carleson measure for $A_{\omega}^{p}$. Moreover,

$$
\left\|G_{\mu, s}^{v}\right\|_{A_{\omega}^{p} \rightarrow L_{\omega}^{q}}^{s} \asymp\left\|I_{d}\right\|_{A_{\omega}^{p} \rightarrow L_{\mu v}^{p+s-\frac{p s}{q}}}^{p+s-\frac{p s}{q}} .
$$

(ii) $G_{\mu, s}^{v}: A_{\omega}^{p} \rightarrow L_{\omega}^{q}$ is compact if and only if $I_{d}: A_{\omega}^{p} \rightarrow L_{\mu_{v}^{\omega}}^{p+s-\frac{p s}{q}}$ is compact.

Theorem 4(i) will be proved in two parts. We first deal with the case $q \geq p$. 
Theorem 5. Let $0<p \leq q<\infty, 0<s<\infty$ and $\omega \in \widehat{\mathcal{D}}$, and let $\mu, v$ be positive Borel measures on $\mathbb{D}$ such that $\mu(\{z \in \mathbb{D}: v(T(z))=0\})=0=\mu(\{0\})$. Then $G_{\mu, s}^{v}: A_{\omega}^{p} \rightarrow L_{\omega}^{q}$ is bounded if and only if $\mu_{v}^{\omega}$ is a $\left(p+s-\frac{p s}{q}\right)$-Carleson measure for $A_{\omega}^{p}$. Moreover,

$$
\left\|G_{\mu, s}^{v}\right\|_{A_{\omega}^{p} \rightarrow L_{\omega}^{q}}^{s} \asymp\left\|I_{d}\right\|_{A_{\omega}^{p} \rightarrow L_{\mu_{v}^{\omega}}^{p+s-\frac{p s}{q}}}^{p+s s} \asymp \sup _{a \in \mathbb{D}} \frac{\mu_{v}^{\omega}(S(a))}{\omega(S(a))^{1+\frac{s}{p}-\frac{s}{q}}} .
$$

Proof. Let first $q>s$. Assume that $G_{\mu, s}^{v}: A_{\omega}^{p} \rightarrow L_{\omega}^{q}$ is bounded. Let $a \in \mathbb{D}$ and choose $\gamma=\gamma(p, q, s)$ sufficiently large so that $\left\|F_{a, p}\right\|_{A_{\omega}^{p}}^{p} \asymp \omega(S(a))$ and $\left\|F_{a,\left(\frac{q}{s}\right)^{\prime}}\right\|_{A_{\omega}^{\left(\frac{q}{s}\right)^{\prime}}}^{\left(\frac{q}{q}\right)^{\prime}} \asymp \omega(S(a))$. Then Fubini's theorem and Hölder's inequality yield

$$
\begin{aligned}
\mu_{v}^{\omega}(S(a)) & \asymp \int_{S(a)}\left|F_{a, p}(z)\right|^{s} d \mu_{v}^{\omega}(z) \\
& \asymp \int_{S(a)}\left|F_{a, p}(z)\right|^{s}\left(\frac{1}{v(T(z))} \int_{T(z)}\left|F_{a,\left(\frac{q}{s}\right)^{\prime}}(\zeta)\right| \omega(\zeta) d A(\zeta)\right) d \mu(z) \\
& \lesssim \int_{\mathbb{D}}\left|F_{a,\left(\frac{q}{s}\right)^{\prime}}(\zeta)\right|\left(\int_{\Gamma(\zeta)}\left|F_{a, p}(z)\right|^{s} \frac{d \mu(z)}{v(T(z))}\right) \omega(\zeta) d A(\zeta) \\
& \leq\left\|F_{a,\left(\frac{q}{s}\right)^{\prime}}\right\|_{A_{\omega}^{\left(\frac{q}{s}\right)^{\prime}}}\left\|G_{\mu, s}^{v}\left(F_{a, p}\right)\right\|_{L_{\omega}^{q}}^{s} \leq\left\|F_{a,\left(\frac{q}{s}\right)^{\prime}}\right\|_{A_{\omega}^{\left(\frac{q}{s}\right)^{\prime}}}\left\|G_{\mu, s}^{v}\right\|_{A_{\omega}^{p} \rightarrow L_{\omega}^{q}}^{s}\left\|F_{a, p}\right\|_{A_{\omega}^{p}}^{s} \\
& \asymp\left\|G_{\mu, s}^{v}\right\|_{A_{\omega}^{p} \rightarrow L_{\omega}^{q}}^{s} \omega(S(a))^{\frac{q}{\left(\frac{q}{)^{\prime}}\right.}} \omega(S(a))^{\frac{s}{p}} \asymp\left\|G_{\mu, s}^{v}\right\|_{A_{\omega}^{p} \rightarrow L_{\omega}^{q}}^{s} \omega(S(a))^{1+\frac{s}{p}-\frac{s}{q}}
\end{aligned}
$$

Hence $\sup _{a \in \mathbb{D}} \frac{\mu_{v}^{\omega}(S(a))}{\omega(S(a))^{1+\frac{s}{p}-\frac{s}{q}}} \lesssim\left\|G_{\mu, s}^{v}\right\|_{A_{\omega}^{p} \rightarrow L_{\omega}^{q}}^{s}$ and $\mu_{v}^{\omega}$ is a $\left(p+s-\frac{p s}{q}\right)$-Carleson measure for $A_{\omega}^{p}$ by Theorem 3 ,

Conversely, let $q>s$ and $\mu_{v}^{\omega}$ be a $\left(p+s-\frac{p s}{q}\right)$-Carleson measure for $A_{\omega}^{p}$. Write $t=$ $t(p, q, s)=p+s-\frac{p s}{q}>s$ for short. Since $\frac{t}{p}=\left(\frac{t}{s}\right)^{\prime} /\left(\frac{q}{s}\right)^{\prime}$, [13, Theorem 3] shows that

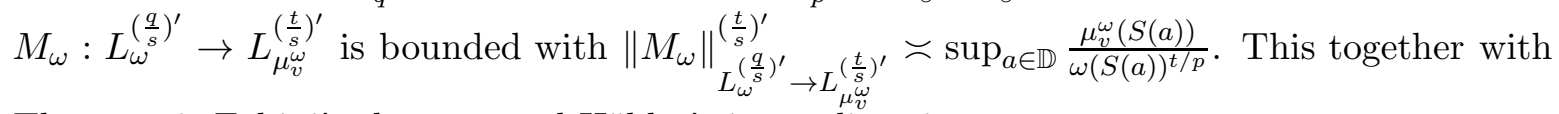
Theorem [3, Fubini's theorem and Hölder's inequality give

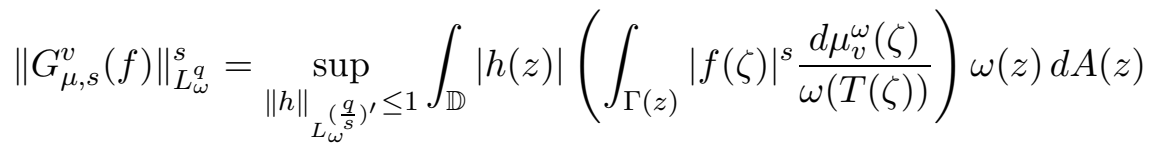

$$
\begin{aligned}
& =\sup _{\|h\|_{L_{\omega}\left(\frac{q}{s}\right)^{\prime}} \leq 1} \int_{\mathbb{D}}|f(\zeta)|^{s}\left(\frac{1}{\omega(T(\zeta))} \int_{T(\zeta)}|h(z)| \omega(z) d A(z)\right) d \mu_{v}^{\omega}(\zeta)
\end{aligned}
$$

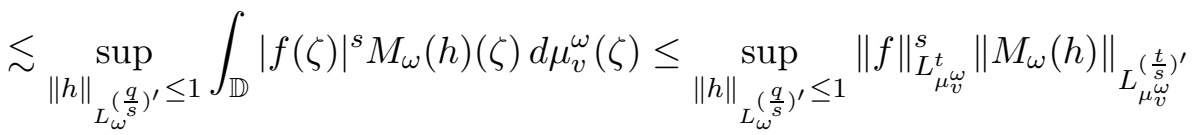

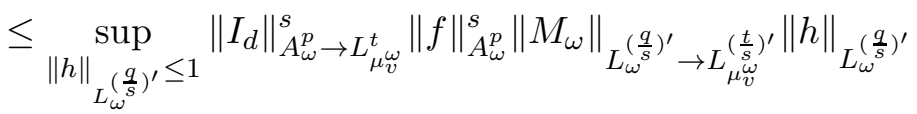

$$
\begin{aligned}
& \asymp\left(\sup _{a \in \mathbb{D}} \frac{\mu_{v}^{\omega}(S(a))}{\omega(S(a))^{1+\frac{s}{p}-\frac{s}{q}}}\right)^{\frac{s}{t}+\frac{1}{\left(\frac{t}{s}\right)^{\prime}}}\|f\|_{A_{\omega}^{p}}^{s}
\end{aligned}
$$

and hence $G_{\mu, s}^{v}: A_{\omega}^{p} \rightarrow L_{\omega}^{q}$ is bounded and $\left\|G_{\mu, s}^{v}(f)\right\|_{L_{\omega}^{q}}^{s} \lesssim \sup _{a \in \mathbb{D}} \frac{\mu_{v}^{\omega}(S(a))}{\omega(S(a))^{1+\frac{s}{p}-\frac{s}{q}}}$.

Since the assertion is valid for $q=s$ by (1.1), it remains to consider the case $q<s$. Let first $\mu_{v}^{\omega}$ be a $\left(p+s-\frac{p s}{q}\right)$-Carleson measure for $A_{\omega}^{p}$, and let $0<x<s$. Hölder's inequality 
and Fubini's theorem yield

$$
\begin{aligned}
\left\|G_{\mu, s}^{v}(f)\right\|_{L_{\omega}^{q}}^{q} & =\int_{\mathbb{D}}\left(\int_{\Gamma(z)}|f(\zeta)|^{x+s-x} \frac{d \mu_{v}^{\omega}(\zeta)}{\omega(T(\zeta))}\right)^{\frac{q}{s}} \omega(z) d A(z) \\
& \leq \int_{\mathbb{D}} N(f)(z)^{\frac{q x}{s}}\left(\int_{\Gamma(z)}|f(\zeta)|^{s-x} \frac{d \mu_{v}^{\omega}(\zeta)}{\omega(T(\zeta))}\right)^{\frac{q}{s}} \omega(z) d A(z) \\
& \leq\left(\int_{\mathbb{D}} N(f)(z)^{\frac{q x}{s-q}} \omega(z) d A(z)\right)^{1-\frac{q}{s}}\left(\int_{\mathbb{D}} \int_{\Gamma(z)}|f(\zeta)|^{s-x} \frac{d \mu_{v}^{\omega}(\zeta)}{\omega(T(\zeta))} \omega(z) d A(z)\right)^{\frac{q}{s}} \\
& \lesssim\|N(f)\|_{L_{\omega}^{\frac{q x}{s-q}}}^{\frac{q q}{s-q}}\left(\int_{\mathbb{D}}|f(\zeta)|^{s-x} d \mu_{v}^{\omega}(\zeta)\right)^{\frac{q}{s}} \asymp\|N(f)\|_{L_{\omega}^{\frac{q x}{s-q}}}^{\frac{q q}{s-q}}\|\|_{L_{\mu_{v}^{(s-x)}}^{\frac{q(s-x)}{s}} .}
\end{aligned}
$$

Take $x=\frac{p(s-q)}{q}<s$ so that $s-x=s+p-\frac{p s}{q}$. Then the estimates above together with [12, Lemma 4.4] and Theorem 3 give

$$
\left\|G_{\mu, s}^{v}(f)\right\|_{L_{\omega}^{q}}^{q} \lesssim\|f\|_{A_{\omega}^{p}}^{\frac{q x}{s}}\left\|I_{d}\right\|_{A_{\omega}^{p} \rightarrow L_{\mu_{v}^{\omega}}^{s+p-\frac{p s}{q}}}^{\frac{q(s-x)}{s}}\|f\|_{A_{\omega}^{p}}^{\frac{q(s-x)}{s}} \asymp\left(\sup _{a \in \mathbb{D}} \frac{\mu_{v}^{\omega}(S(a))}{\omega(S(a))^{1+\frac{s}{p}-\frac{s}{q}}}\right)^{\frac{q}{s}}\|f\|_{A_{\omega}^{p}}^{q},
$$

and hence $G_{\mu, s}^{v}: A_{\omega}^{p} \rightarrow L_{\omega}^{q}$ is bounded with $\left\|G_{\mu}^{v}\right\|_{A_{\omega}^{p} \rightarrow L_{\omega}^{q}}^{s} \lesssim \sup _{a \in \mathbb{D}} \frac{\mu_{v}^{\omega}(S(a))}{\omega(S(a))^{1+\frac{s}{p}-\frac{s}{q}}}$.

Conversely, let $q<s$ and $G_{\mu, s}^{v}: A_{\omega}^{p} \rightarrow L_{\omega}^{q}$ be bounded. Choose $\alpha>\beta>1$ such that $\frac{\beta}{\alpha}=\frac{q}{s}$. Fubini's theorem and Hölder's inequality yield

$$
\begin{aligned}
& \mu_{v}^{\omega}(S(a))= \int_{\mathbb{D}} \chi_{S(a)}(z) d \mu_{v}^{\omega}(z) \asymp \int_{\mathbb{D}} \chi_{S(a)}(z)\left|F_{a, p}(z)\right|^{s} \frac{1}{v(T(z))} \int_{T(z)} \omega(\zeta) d A(\zeta) d \mu(z) \\
&= \int_{\mathbb{D}}\left(\int_{\Gamma(\zeta)} \chi_{S(a)}(z)\left|F_{a, p}(z)\right|^{s} \frac{d \mu(z)}{v(T(z))}\right)^{\frac{1}{\alpha}+\frac{1}{\alpha^{\prime}}} \omega(\zeta) d A(\zeta) \\
& \leq\left(\int_{\mathbb{D}}\left(\int_{\Gamma(\zeta)}\left|F_{a, p}(z)\right|^{s} \frac{d \mu(z)}{v(T(z))}\right)^{\frac{\beta}{\alpha}} \omega(\zeta) d A(\zeta)\right)^{\frac{1}{\beta}} \\
& \cdot\left(\int_{\mathbb{D}}\left(\int_{\Gamma(\zeta)} \chi_{S(a)}(z) \frac{d \mu(z)}{v(T(z))}\right)^{\frac{\beta^{\prime}}{\alpha^{\prime}}} \omega(\zeta) d A(\zeta)\right)^{\frac{1}{\beta^{\prime}}} \\
&=\left\|G_{\mu, s}^{v}\left(F_{a, p}\right)\right\|_{L_{\omega}^{q}}^{\frac{q}{\beta}}\left\|G_{\mu, 1}^{v}\left(\chi_{S(a)}\right)\right\|^{\frac{1}{\alpha^{\prime}}} \\
& L_{\omega}^{\frac{\beta^{\prime}}{\alpha^{\prime}}}
\end{aligned}
$$


Now $\frac{\beta^{\prime}}{\alpha^{\prime}}>1$ because $\beta<\alpha$, and hence $\left\|G_{\mu, 1}^{v}\left(\chi_{S(a)}\right)\right\|_{L_{\omega}^{\frac{\beta^{\prime}}{\alpha^{\prime}}}}$ can be estimated by duality arguments. Namely, since $\left(\frac{\beta^{\prime}}{\alpha^{\prime}}\right)^{\prime}=\frac{\beta(\alpha-1)}{\alpha-\beta}$, Fubini's theorem, Hölder's inequality and [13, Theorem 3] give

$$
\begin{aligned}
& \left\|G_{\mu, 1}^{v}\left(\chi_{S(a)}\right)\right\|_{L_{\omega}^{\frac{\beta^{\prime}}{\alpha^{\prime}}}}=\sup _{\|h\|_{L_{\omega} \frac{\beta(\alpha-1)}{\alpha-\beta}} \leq 1} \int_{\mathbb{D}}|h(\zeta)| G_{\mu, 1}^{v}\left(\chi_{S(a)}\right)(\zeta) \omega(\zeta) d A(\zeta) \\
& =\sup _{\|h\|} \int_{\frac{\beta(\alpha-1)}{L_{\omega}} \leq 1}|h(\zeta)|\left(\int_{\Gamma(\zeta)} \chi_{S(a)}(z) \frac{d \mu(z)}{v(T(z))}\right) \omega(\zeta) d A(\zeta) \\
& =\sup _{\|h\|} \int_{\frac{\beta(\alpha-1)}{L_{\omega}} \leq 1} \chi_{\mathbb{D}} \chi_{S(a)}(z)\left(\frac{1}{v(T(z))} \int_{T(z)}|h(\zeta)| \omega(\zeta) d A(\zeta)\right) d \mu(z) \\
& =\sup _{\|h\|} \int_{\frac{\beta(\alpha-1)}{L_{\omega}} \leq 1} \int_{\mathbb{D}} \chi_{S(a)}(z)\left(\frac{1}{\omega(T(z))} \int_{T(z)}|h(\zeta)| \omega(\zeta) d A(\zeta)\right) d \mu_{v}^{\omega}(z) \\
& \lesssim_{\|h\|} \sup _{\frac{\beta(\alpha-1)}{L_{\omega}} \leq 1} \int_{\mathbb{D}} \chi_{S(a)}(z) M_{\omega}(h)(z) d \mu_{v}^{\omega}(z) \\
& \leq \sup _{\|h\| \sin _{L_{\omega}(\alpha-\beta)} \leq 1}\left(\int_{\mathbb{D}} \chi_{S(a)}(z) d \mu_{v}^{\omega}(z)\right)^{\frac{\alpha^{\prime}}{\beta^{\prime}}} \\
& \cdot\left(\int_{\mathbb{D}} M_{\omega}(h)(z)^{\frac{\beta(\alpha-1)}{\alpha-\beta}} \chi_{S(a)}(z) d \mu_{v}^{\omega}(z)\right)^{1-\frac{\alpha^{\prime}}{\beta^{\prime}}} \\
& \lesssim \mu_{v}^{\omega}(S(a))^{\frac{\alpha^{\prime}}{\beta^{\prime}}}\left(\sup _{z \in \mathbb{D}} \frac{\mu_{v}^{\omega}(S(z) \cap S(a))}{\omega(S(z))}\right)^{1-\frac{\alpha^{\prime}}{\beta^{\prime}}}, \quad a \in \mathbb{D} \text {. }
\end{aligned}
$$

By combining this with (3.1) and using the norm estimate $\left\|F_{a, p}\right\|_{A_{\omega}^{p}}^{p} \asymp \omega(S(a))$, we deduce

$$
\begin{aligned}
\mu_{v}^{\omega}(S(a)) & \lesssim\left\|G_{\mu, s}^{v}\left(F_{a, p}\right)\right\|_{L_{\omega}^{q}}^{\frac{q}{\beta}}\left\|G_{\mu, 1}^{v}\left(\chi_{S(a)}\right)\right\|_{L_{\omega}^{\frac{\beta^{\prime}}{\alpha^{\prime}}}}^{\frac{1}{\alpha^{\prime}}} \\
& \lesssim\left\|G_{\mu, s}^{v}\right\|_{A_{\omega}^{p} \rightarrow L_{\omega}^{q}}^{\frac{q}{\beta}} \omega(S(a))^{\frac{q}{p \beta}} \mu_{v}^{\omega}(S(a))^{\frac{1}{\beta^{\prime}}}\left(\sup _{z \in \mathbb{D}} \frac{\mu_{v}^{\omega}(S(z) \cap S(a))}{\omega(S(z))}\right)^{\frac{1}{\alpha^{\prime}}-\frac{1}{\beta^{\prime}}},
\end{aligned}
$$

which yields

$$
\mu_{v}^{\omega}(S(a))^{\frac{1}{\beta}} \omega(S(a))^{-\frac{q}{p \beta}} \lesssim\left\|G_{\mu, s}^{v}\right\|_{A_{\omega}^{p} \rightarrow L_{\omega}^{q}}^{\frac{q}{\beta}}\left(\sup _{z \in \mathbb{D}} \frac{\mu_{v}^{\omega}(S(z) \cap S(a))}{\omega(S(z))}\right)^{\frac{1}{\alpha^{\prime}}-\frac{1}{\beta^{\prime}}}, \quad a \in \mathbb{D} .
$$

Define $d \mu_{r}(z)=\chi_{D(0, r)}(z) d \mu(z)$ for $0<r<1$. Then

$$
\begin{aligned}
\left(G_{\mu_{r}, s}^{v}(f)(z)\right)^{s} & =\int_{\Gamma(z)}|f(\zeta)|^{s} \frac{d \mu_{r}(\zeta)}{v(T(\zeta))}=\int_{\Gamma(z) \cap D(0, r)}|f(\zeta)|^{s} \frac{d \mu(\zeta)}{v(T(\zeta))} \\
& \leq \int_{\Gamma(z)}|f(\zeta)|^{s} \frac{d \mu(\zeta)}{v(T(\zeta))}=\left(G_{\mu, s}^{v}(f)(z)\right)^{s}, \quad z \in \mathbb{D} \backslash\{0\},
\end{aligned}
$$

and hence $\left\|G_{\mu_{r}, s}^{v}\right\|_{A_{\omega}^{p} \rightarrow L_{\omega}^{q}} \leq\left\|G_{\mu, s}^{v}\right\|_{A_{\omega}^{p} \rightarrow L_{\omega}^{q}}$ for all $0<r<1$. 
If $q=p$, then (3.3) applied to $\mu_{r}$ implies

$$
\left(\mu_{v}^{\omega}\right)_{r}(S(a))^{\frac{1}{\beta}} \omega(S(a))^{-\frac{1}{\beta}} \lesssim\left\|G_{\mu_{r}, s}^{v}\right\|_{A_{\omega}^{p} \rightarrow L_{\omega}^{p}}^{\frac{p}{\beta}}\left(\sup _{z \in \mathbb{D}} \frac{\left(\mu_{v}^{\omega}\right)_{r}(S(z))}{\omega(S(z))}\right)^{\frac{1}{\alpha^{\prime}}-\frac{1}{\beta^{\prime}}}, \quad a \in \mathbb{D},
$$

and hence

$$
\left(\sup _{z \in \mathbb{D}} \frac{\left(\mu_{v}^{\omega}\right)_{r}(S(z))}{\omega(S(z))}\right)^{\frac{1}{\beta}} \lesssim\left\|G_{\mu, s}^{v}\right\|_{A_{\omega}^{p} \rightarrow L_{\omega}^{p}}^{\frac{p}{\beta}}\left(\sup _{z \in \mathbb{D}} \frac{\left(\mu_{v}^{\omega}\right)_{r}(S(z))}{\omega(S(z))}\right)^{\frac{1}{\alpha^{\prime}}-\frac{1}{\beta^{\prime}}} .
$$

Consequently,

$$
\sup _{z \in \mathbb{D}, r \in(0,1)} \frac{\left(\mu_{v}^{\omega}\right)_{r}(S(z))}{\omega(S(z))} \lesssim\left\|G_{\mu, s}^{v}\right\|_{A_{\omega}^{p} \rightarrow L_{\omega}^{p}}^{s}
$$

So Fatou's lemma and Theorem 3 show that $\mu_{v}^{\omega}$ is a $p$-Carleson measure for $A_{\omega}^{p}$ with

$$
\left\|I_{d}\right\|_{A_{\omega}^{p} \rightarrow L_{\mu_{v}^{\omega}}^{p}} \asymp \sup _{a \in \mathbb{D}} \frac{\mu_{v}^{\omega}(S(a))}{\omega(S(a))} \lesssim\left\|G_{\mu, s}^{v}\right\|_{A_{\omega}^{p} \rightarrow L_{\omega}^{p}}^{s}
$$

If $q>p$, then applying (3.3) to $\mu_{r}$ and bearing in mind that $\omega$ is radial

$$
\begin{aligned}
\frac{\left(\mu_{v}^{\omega}\right)_{r}(S(a))}{\omega(S(a))^{1+\frac{s}{p}-\frac{s}{q}}} & \lesssim\left\|G_{\mu_{r}, s}^{v}\right\|_{A_{\omega}^{p} \rightarrow L_{\omega}^{q}}^{q} \omega(S(a))^{\frac{q}{p}-1-\frac{s}{p}+\frac{s}{q}}\left(\sup _{z \in \mathbb{D}} \frac{\left(\mu_{v}^{\omega}\right)_{r}(S(z) \cap S(a))}{\omega(S(z))}\right)^{\frac{\beta}{\alpha^{\prime}}-\frac{\beta}{\beta^{\prime}}} \\
& =\left\|G_{\mu_{r}, s}^{v}\right\|_{A_{\omega}^{p} \rightarrow L_{\omega}^{q}}^{q} \omega(S(a))^{\frac{-(q-p)(s-q)}{p q}}\left(\sup _{z: S(z) \subset S(a)} \frac{\left(\mu_{v}^{\omega}\right)_{r}(S(z) \cap S(a))}{\omega(S(z))}\right)^{1-\frac{q}{s}} \\
& =\left\|G_{\mu_{r}, s}^{v}\right\|_{A_{\omega}^{p} \rightarrow L_{\omega}^{q}}^{q}\left(\sup _{z: S(z) \subset S(a)} \frac{\left(\mu_{v}^{\omega}\right)_{r}(S(z) \cap S(a))}{\omega(S(a))^{s \frac{q-p}{p q}} \omega(S(z))}\right)^{1-\frac{q}{s}} \\
& \leq\left\|G_{\mu_{r}, s}^{v}\right\|_{A_{\omega}^{p} \rightarrow L_{\omega}^{q}}^{q}\left(\sup _{z: S(z) \subset S(a)} \frac{\left(\mu_{v}^{\omega}\right)_{r}(S(z))}{\omega(S(z))^{1+\frac{s}{p}-\frac{s}{q}}}\right)^{1-\frac{q}{s}}, \quad a \in \mathbb{D} .
\end{aligned}
$$

Consequently,

$$
\begin{aligned}
\sup _{a \in \mathbb{D}}\left(\frac{\left(\mu_{v}^{\omega}\right)_{r}(S(a))}{\omega(S(a))^{1+\frac{s}{p}-\frac{s}{q}}}\right) & \lesssim\left\|G_{\mu, s}^{v}\right\|_{A_{\omega}^{p} \rightarrow L_{\omega}^{q}}^{q}\left(\sup _{a \in \mathbb{D}} \sup _{z: S(z) \subset S(a)} \frac{\left(\mu_{v}^{\omega}\right)_{r}(S(z))}{\omega(S(z))^{1+\frac{s}{p}-\frac{s}{q}}}\right)^{1-\frac{q}{s}} \\
& =\left\|G_{\mu, s}^{v}\right\|_{A_{\omega}^{p} \rightarrow L_{\omega}^{q}}^{q}\left(\sup _{a \in \mathbb{D}} \frac{\left(\mu_{v}^{\omega}\right)_{r}(S(a))}{\omega(S(a))^{1+\frac{s}{p}-\frac{s}{q}}}\right)^{1-\frac{q}{s}}
\end{aligned}
$$

and thus

$$
\sup _{a \in \mathbb{D}, r \in(0,1)} \frac{\left(\mu_{v}^{\omega}\right)_{r}(S(a))}{\omega(S(a))^{1+\frac{s}{p}-\frac{s}{q}}} \lesssim\left\|G_{\mu, s}^{v}\right\|_{A_{\omega}^{p} \rightarrow L_{\omega}^{q}}^{s}
$$

Fatou's lemma and Theorem 3 show that $\mu_{v}^{\omega}$ is a $\left(p+s-\frac{p s}{q}\right)$-Carleson measure for $A_{\omega}^{p}$ with the corresponding inequality of norms.

Theorem 6. Let $0<q<p<\infty$ and $0<s<\infty$ such that $1+\frac{s}{p}-\frac{s}{q}>0, \omega \in \widehat{\mathcal{D}}$ and let $\mu, v$ be positive Borel measures on $\mathbb{D}$ such that $\mu(\{z \in \mathbb{D}: v(T(z))=0\})=0=\mu(\{0\})$. Then $G_{\mu, s}^{v}: A_{\omega}^{p} \rightarrow L_{\omega}^{q}$ is bounded if and only if $\mu_{v}^{\omega}$ is a $p\left(1+\frac{s}{p}-\frac{s}{q}\right)$-Carleson measure for $A_{\omega}^{p}$. Moreover,

$$
\left\|G_{\mu, s}^{v}\right\|_{A_{\omega}^{p} \rightarrow L_{\omega}^{q}}^{s} \asymp\left\|I_{d}\right\|_{A_{\omega}^{p} \rightarrow L_{\mu_{v}^{\omega}}^{p+\frac{p-\frac{p s}{q}}{q}}}^{p} \asymp\left\|B_{\mu}^{v}\right\|_{L_{\omega}^{\frac{q p}{s(p-q)}}} .
$$


Proof. The equivalence $\left\|I_{d}\right\|_{A_{\omega}^{p} \rightarrow L_{\mu_{v}^{\omega}}^{p+s-\frac{p s}{q}}}^{p+\frac{p s}{q}} \asymp\left\|B_{\mu}^{v}\right\|_{L_{\omega}^{\frac{q p}{s(p-q)}}}$ follows from Theorem 3 , If $B_{\mu}^{v} \in L_{\omega}^{\frac{q p}{s(p-q)}}$, then Hölder's inequality and [12, Lemma 4.4] give

$$
\begin{aligned}
\left\|G_{\mu, s}^{v}(f)\right\|_{L_{\omega}^{q}}^{q} & =\int_{\mathbb{D}}\left(\int_{\Gamma(z)}|f(\zeta)|^{s} \frac{d \mu(\zeta)}{v(T(\zeta))}\right)^{\frac{q}{s}} \omega(z) d A(z) \\
& \leq \int_{\mathbb{D}} N(f)^{q}(z)\left(\int_{\Gamma(z)} \frac{d \mu(\zeta)}{v(T(\zeta))}\right)^{\frac{q}{s}} \omega(z) d A(z) \\
& \leq\|N(f)\|_{L_{\omega}^{p}}^{q}\left\|B_{\mu}^{v}\right\|_{L_{\omega}^{\frac{q}{s}}}^{\frac{q p}{s(p-q)}} \asymp\|f\|_{A_{\omega}^{p}}^{q}\left\|B_{\mu}^{v}\right\|_{L_{\omega}^{\frac{q}{s}}}^{\frac{q}{s(p-q)}},
\end{aligned}
$$

and hence $G_{\mu, s}^{v}: A_{\omega}^{p} \rightarrow L_{\omega}^{q}$ is bounded and $\left\|G_{\mu, s}^{v}\right\|_{A_{\omega}^{p} \rightarrow L_{\omega}^{q}}^{s} \lesssim\left\|B_{\mu}^{v}\right\|_{L_{\omega}^{\frac{q p}{p-q}}}$.

Assume now that $G_{\mu, s}^{v}: A_{\omega}^{p} \rightarrow L_{\omega}^{q}$ is bounded, and let first $q>s$ and write $t=t(p, q, s)=$ $\left(p+s-\frac{p s}{q}\right)=s+p\left(1-\frac{s}{q}\right)$. It suffices to show that $\mu_{v}^{\omega}$ is a $t$-Carleson measure for $A_{\omega}^{p}$. Fubini's theorem, Hölder's inequality and [12, Lemma 4.4] yield

$$
\begin{aligned}
\|f\|_{L_{\mu_{v}}^{t}}^{t} & =\int_{\mathbb{D}}|f(z)|^{t}\left(\int_{T(z)} \omega(\zeta) d A(\zeta)\right) \frac{d \mu(z)}{v(T(z))} \\
& =\int_{\mathbb{D}}\left(\int_{\Gamma(\zeta)}|f(z)|^{s+p\left(1-\frac{s}{q}\right)} \frac{d \mu(z)}{v(T(z))}\right) \omega(\zeta) d A(\zeta) \\
& \leq \int_{\mathbb{D}} N(f)^{p\left(1-\frac{s}{q}\right)}(\zeta)\left(\int_{\Gamma(\zeta)}|f(z)|^{s} \frac{d \mu(z)}{v(T(z))}\right) \omega(\zeta) d A(\zeta) \\
& \lesssim\|N(f)\|_{L_{\omega}^{p}}^{p\left(1-\frac{s}{q}\right)}\left\|G_{\mu, s}^{v}(f)\right\|_{L_{\omega}^{q}}^{s} \asymp\|f\|_{A_{\omega}^{p}}^{p\left(1-\frac{s}{q}\right)}\left\|G_{\mu, s}^{v}(f)\right\|_{L_{\omega}^{q}}^{s} \\
& \leq\left\|G_{\mu}^{v}\right\|_{A_{\omega}^{p} \rightarrow L_{\omega}^{q}\|f\|_{A_{\omega}^{p}}^{s}}
\end{aligned}
$$

and hence $I_{d}: A_{\omega}^{p} \rightarrow L_{\mu_{v}^{\omega}}^{t}$ is bounded with $\left\|I_{d}\right\|_{A_{\omega}^{p} \rightarrow L_{\mu_{v}^{\omega}}^{t}}^{t} \lesssim\left\|G_{\mu}^{v}\right\|_{A_{\omega}^{p} \rightarrow L_{\omega}^{q}}^{s}$.

If $q=s$, then $t=s$ and the result follows from (1.1).

If $q<s$, then $t=p+s-\frac{p s}{q}<s$ and $\frac{q}{t}>1$ because $\min \{p, s\}>q$. Hence Hölder's inequality gives

$$
\begin{aligned}
& \|f\|_{L_{\mu_{v}}^{t}}^{t}=\int_{\mathbb{D}}\left(\int_{\Gamma(\zeta)}|f(z)|^{t} \frac{d \mu(z)}{v(T(z))}\right) \omega(\zeta) d A(\zeta) \\
& \lesssim \int_{\mathbb{D}}\left(\int_{\Gamma(\zeta)}|f(z)|^{s} \frac{d \mu(z)}{v(T(z))}\right)^{\frac{t}{s}}\left(\int_{\Gamma(\zeta)} \frac{d \mu(z)}{v(T(z))}\right)^{1-\frac{t}{s}} \omega(\zeta) d A(\zeta) \\
& \leq\left(\int_{\mathbb{D}}\left(\int_{\Gamma(\zeta)}|f(z)|^{s} \frac{d \mu(z)}{v(T(z))}\right)^{\frac{q}{s}} \omega(\zeta) d A(\zeta)\right)^{\frac{t}{q}} \\
& \cdot\left(\int_{\mathbb{D}}\left(\int_{\Gamma(\zeta)} \frac{d \mu(z)}{v(T(z))}\right)^{\frac{s-t}{s} \frac{q}{q-t}} \omega(\zeta) d A(\zeta)\right)^{\frac{q-t}{q}}
\end{aligned}
$$

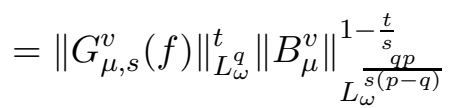

$$
\begin{aligned}
& \leq\left\|G_{\mu, s}^{v}\right\|_{A_{\omega}^{p} \rightarrow L_{\omega}^{q}}^{t}\left\|B_{\mu, s}^{v}\right\|_{L_{\omega}^{\frac{q p}{s(p-q)}}}^{1-\frac{t}{s}}\|f\|_{A_{\omega}^{p}}^{t} .
\end{aligned}
$$


This applied to $\mu_{r}$ yields

$$
\left(\frac{\|f\|_{L_{\left(\mu_{v}^{\omega}\right)_{r}}^{t}}}{\|f\|_{A_{\omega}^{p}}^{p}}\right)^{t} \lesssim\left\|G_{\mu_{r}, s}^{v}\right\|_{A_{\omega}^{p} \rightarrow L_{\omega}^{q}}^{t}\left\|B_{\mu_{r}}^{v}\right\|_{L_{\omega}^{\frac{q}{s(p-q)}}}^{1-\frac{t}{s}}, \quad f \in A_{\omega}^{p}, \quad f \not \equiv 0
$$

and hence

$$
\left\|I_{d}\right\|_{A_{\omega}^{p} \rightarrow L_{\left(\mu_{v}\right)_{r}}^{t}}^{t} \leq\left\|G_{\mu_{r}, s}^{v}\right\|_{A_{\omega}^{p} \rightarrow L_{\omega}^{q}}^{t}\left\|B_{\mu_{r}}^{v}\right\|_{L_{\omega}^{\frac{q}{s(p-q)}}}^{1-\frac{t}{s}} .
$$

Since $\left\|I_{d}\right\|_{A_{\omega}^{p} \rightarrow L_{\left(\mu_{v}^{\omega}\right)_{r}}^{t}}^{t} \asymp\left\|B_{\mu_{r}}^{v}\right\|_{L_{\omega}^{\frac{q p}{s(p-q)}}}$ by Theorem 3, we deduce

$$
\left\|B_{\mu_{r}}^{v}\right\|_{L_{\omega}^{\frac{q p}{s(p-q)}}} \lesssim\left\|G_{\mu_{r}, s}^{v}\right\|_{A_{\omega}^{p} \rightarrow L_{\omega}^{q}}^{t}\left\|B_{\mu_{r}}^{v}\right\|_{L_{\omega}^{\frac{s^{q}}{s(p-q)}}}^{1-\frac{t}{s}},
$$

and hence

$$
\left\|B_{\mu_{r}}^{v}\right\|_{L_{\omega}^{\frac{q p}{s(p-q)}}} \lesssim\left\|G_{\mu_{r}, s}^{v}\right\|_{A_{\omega}^{p} \rightarrow L_{\omega}^{q}}^{s} \leq\left\|G_{\mu, s}^{v}\right\|_{A_{\omega}^{p} \rightarrow L_{\omega}^{q}}^{s},
$$

which together with Fatou's lemma gives

$$
\left\|B_{\mu}^{v}\right\|_{L_{\omega}^{\frac{q p}{s(p-q)}}} \leq \liminf _{r \rightarrow 1}\left\|B_{\mu_{r}}^{v}\right\|_{L_{\omega} \frac{q p}{s(p-q)}} \lesssim\left\|G_{\mu, s}^{v}\right\|_{A_{\omega}^{p} \rightarrow L_{\omega}^{q}}^{s} .
$$

This finishes the proof.

\section{Compact operators $G_{\mu, s}^{v}: A_{\omega}^{p} \rightarrow L_{\omega}^{q}$}

In this section we prove Theorem 4 (ii).

Lemma 7. Let $\nu$ be a finite positive Borel measure on $\mathbb{D}$ and $0<p<\infty$. If $\left\{\varphi_{n}\right\}_{n=0}^{\infty} \subset L_{\nu}^{p}$ and $\varphi \in L_{\nu}^{p}$ satisfy $\lim _{n \rightarrow \infty}\left\|\varphi_{n}\right\|_{L_{\nu}^{p}}=\|\varphi\|_{L_{\nu}^{p}}$ and $\lim _{n \rightarrow \infty} \varphi_{n}(z)=\varphi(z) \nu$-a.e. on $\mathbb{D}$, then $\lim _{n \rightarrow \infty}\left\|\varphi_{n}-\varphi\right\|_{L_{\nu}^{p}}=0$.

Proof. See the proof of [5, Lemma 1 p. 21].

Theorem 8. Let $0<p \leq q<\infty, 0<s<\infty$ and $\omega \in \widehat{\mathcal{D}}$, and let $\mu, v$ be positive Borel measures on $\mathbb{D}$ such that $\mu(\{z \in \mathbb{D}: v(T(z))=0\})=0=\mu(\{0\})$. Then $G_{\mu, s}^{v}: A_{\omega}^{p} \rightarrow L_{\omega}^{q}$ is compact if and only if $I_{d}: A_{\omega}^{p} \rightarrow L_{\mu_{v}^{\omega}}^{p+s-\frac{p s}{q}}$ is compact.

Proof. Let first $q>s$. Assume that $G_{\mu, s}^{v}: A_{\omega}^{p} \rightarrow L_{\omega}^{q}$ is compact. For each $a \in \mathbb{D}$, let $f_{a, p}(z)=$ $(\omega(S(a)))^{-\frac{1}{p}} F_{a, p}(z)=(\omega(S(a)))^{-\frac{1}{p}}\left(\frac{1-|a|^{2}}{1-\bar{a} z}\right)^{\frac{\gamma+1}{p}}$. By Lemma B we may choose $\gamma=\gamma(p, q, s, \omega)$ sufficiently large such that $\sup _{a \in \mathbb{D}}\left\|f_{a, p}\right\|_{A_{\omega}^{p}}^{p} \asymp 1, \sup _{a \in \mathbb{D}}\left\|f_{a,\left(\frac{q}{s}\right)^{\prime}}\right\|_{A_{\omega}^{\left(\frac{q}{s}\right)^{\prime}}}^{\left(\frac{q}{s}\right)^{\prime}} \asymp 1$ and $f_{a, p}$ converges uniformly to zero on compact subsets of $\mathbb{D}$ as $|a| \rightarrow 1^{-}$. A standard argument shows that

$$
\lim _{|a| \rightarrow 1^{-}}\left\|G_{\mu, s}^{v}\left(f_{a, p}\right)\right\|_{L_{\omega}^{q}}=0 .
$$

Fubini's theorem and Hölder's inequality yield

$$
\begin{aligned}
\frac{\mu_{v}^{\omega}(S(a))}{\omega(S(a))^{1+\frac{s}{p}-\frac{s}{q}}} & \asymp \frac{1}{\omega(S(a))^{1-\frac{s}{q}}} \int_{S(a)}\left|f_{a, p}(z)\right|^{s} d \mu_{v}^{\omega}(z) \\
& \asymp \int_{S(a)}\left|f_{a, p}(z)\right|^{s}\left(\frac{1}{v(T(z))} \int_{T(z)}\left|f_{a,\left(\frac{q}{s}\right)^{\prime}}(\zeta)\right| \omega(\zeta) d A(\zeta)\right) d \mu(z) \\
& \lesssim \int_{\mathbb{D}}\left|f_{a,\left(\frac{q}{s}\right)^{\prime}}(\zeta)\right|\left(\int_{\Gamma(\zeta)}\left|f_{a, p}(z)\right|^{s} \frac{d \mu(z)}{v(T(z))}\right) \omega(\zeta) d A(\zeta) \\
& \leq\left\|f_{a,\left(\frac{q}{s}\right)^{\prime}}\right\|_{A_{\omega}^{\left(\frac{q}{s}\right)^{\prime}}}\left\|G_{\mu, s}^{v}\left(f_{a, p}\right)\right\|_{L_{\omega}^{q}}^{s} \lesssim\left\|G_{\mu, s}^{v}\left(f_{a, p}\right)\right\|_{L_{\omega}^{q}}^{s}
\end{aligned}
$$


This together with (4.1) gives $\lim _{|a| \rightarrow 1^{-}} \frac{\mu_{v}^{\omega}(S(a))}{\omega(S(a))^{1+\frac{s}{p}-\frac{s}{q}}}=0$ and hence $I_{d}: A_{\omega}^{p} \rightarrow L_{\mu_{v}^{\omega}}^{p+s-\frac{p s}{q}}$ is compact by Theorem 3 .

Conversely, let $q>s$ and assume that $I_{d}: A_{\omega}^{p} \rightarrow L_{\mu_{v}^{\omega}}^{p+s-\frac{p s}{q}}$ is compact. Write $t=t(p, q, s)=$ $p+s-\frac{p s}{q}>s$ for short. Let $\left\{f_{n}\right\}$ be a bounded sequence in $A_{\omega}^{p}$. Then, we may extract a subsequence $\left\{f_{n_{k}}\right\}$ that converges in $L_{\mu_{v}^{\omega}}^{t}$ and uniformly on compact subsets to some $f \in A_{\omega}^{p}$. Write $g_{n_{k}}=f_{n_{k}}-f$. By Fubini's theorem, [13, Theorem 3] and Hölder's inequality,

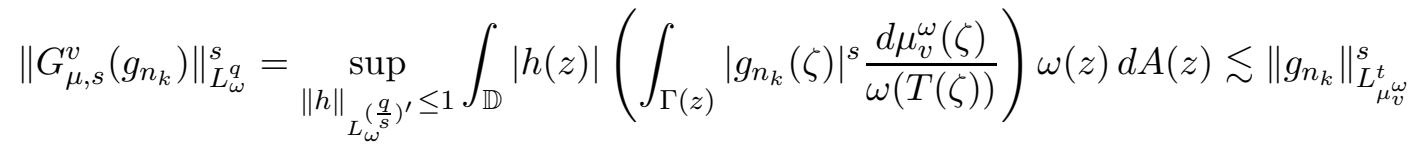

and hence

$$
\lim _{k \rightarrow \infty}\left\|G_{\mu, s}^{v}\left(g_{n_{k}}\right)\right\|_{L_{\omega}^{q}}^{s}=0 .
$$

If $s \geq 1$, two applications of Minkowski's inequality gives

$$
\left|\left\|G_{\mu, s}^{v}\left(f_{n_{k}}\right)\right\|_{L_{\omega}^{q}}-\left\|G_{\mu, s}^{v}(f)\right\|_{L_{\omega}^{q}}\right| \leq\left\|G_{\mu, s}^{v}\left(g_{n_{k}}\right)\right\|_{L_{\omega}^{q}} \rightarrow 0, \quad k \rightarrow \infty .
$$

Moreover, since $\left\{f_{n_{k}}\right\}$ converges uniformly on compact subsets of $\mathbb{D}$ to $f$, then $\varphi_{k}(z)=$ $\left(\int_{\Gamma(z)}\left|f_{n_{k}}(\zeta)\right|^{s} \frac{d \mu_{v}^{\omega}(\zeta)}{\omega(T(\zeta))}\right)^{1 / s}$ converges to $\varphi(z)=\left(\int_{\Gamma(z)}|f(\zeta)|^{s} \frac{d \mu_{v}^{\omega}(\zeta)}{\omega(T(\zeta))}\right)^{1 / s}$ for each $z \in \mathbb{D}$. Therefore Lemma 7 yields

$$
\lim _{k \rightarrow \infty}\left\|G_{\mu, s}^{v}\left(f_{n_{k}}\right)-G_{\mu, s}^{v}(f)\right\|_{L_{\omega}^{q}}=\lim _{k \rightarrow \infty}\left\|\varphi_{k}-\varphi\right\|_{L_{\omega}^{q}}=0,
$$

and thus $G_{\mu, s}^{v}: A_{\omega}^{p} \rightarrow L_{\omega}^{q}$ is compact.

If $0<s \leq 1$,

$$
\int_{\Gamma(z)}\left|f_{n_{k}}(\zeta)\right|^{s} \frac{d \mu_{v}^{\omega}(\zeta)}{\omega(T(\zeta))} \leq \int_{\Gamma(z)}\left|g_{n_{k}}(\zeta)\right|^{s} \frac{d \mu_{v}^{\omega}(\zeta)}{\omega(T(\zeta))}+\int_{\Gamma(z)}|f(\zeta)|^{s} \frac{d \mu_{v}^{\omega}(\zeta)}{\omega(T(\zeta))}
$$

which together with Minkowski's inequality yields

$$
\left|\left\|G_{\mu, s}^{v}\left(f_{n_{k}}\right)\right\|_{L_{\omega}^{q}}^{s}-\left\|G_{\mu, s}^{v}(f)\right\|_{L_{\omega}^{q}}^{s}\right| \leq\left\|G_{\mu, s}^{v}\left(g_{n_{k}}\right)\right\|_{L_{\omega}^{q}}^{s} .
$$

Now, by arguing as in the previous case we see that $G_{\mu, s}^{v}: A_{\omega}^{p} \rightarrow L_{\omega}^{q}$ is compact. In view of (1.1), a similar reasoning also applies in the case $q=s$.

It remains to consider the case $q<s$. Assume first that $I_{d}: A_{\omega}^{p} \rightarrow L_{\mu_{v}^{\omega}}^{p+s-\frac{p s}{q}}$ is compact. Let $\left\{f_{n}\right\}$ be a bounded sequence in $A_{\omega}^{p}$. Then we may extract a subsequence $\left\{f_{n_{k}}\right\}$ that converges on $L_{\mu_{v}^{\omega}}^{p+s-\frac{p s}{q}}$ and uniformly on compact subsets of $\mathbb{D}$ to some $f \in A_{\omega}^{p}$. Write $g_{n_{k}}=f_{n_{k}}-f$, and let $0<x<s$. Hölder's inequality and Fubini's theorem yield

$$
\begin{aligned}
\left\|G_{\mu, s}^{v}\left(g_{n_{k}}\right)\right\|_{L_{\omega}^{q}}^{q} & \leq \int_{\mathbb{D}} N\left(g_{n_{k}}\right)(z)^{\frac{q x}{s}}\left(\int_{\Gamma(z)}\left|g_{n_{k}}(\zeta)\right|^{s-x} \frac{d \mu_{v}^{\omega}(\zeta)}{\omega(T(\zeta))}\right)^{\frac{q}{s}} \omega(z) d A(z) \\
& \lesssim\left\|N\left(g_{n_{k}}\right)\right\|_{L_{\omega}^{\frac{q x}{s-q}}}^{\frac{q x}{s-q}}\left\|g_{n_{k}}\right\|_{L_{\mu_{v}}^{(s-x)}}^{\frac{q(s-x)}{s-x}} .
\end{aligned}
$$

Take $x=\frac{p(s-q)}{q}<s$ so that $s-x=s+p-\frac{p s}{q}$. Then the estimates above together with [12, Lemma 4.4] give

and hence

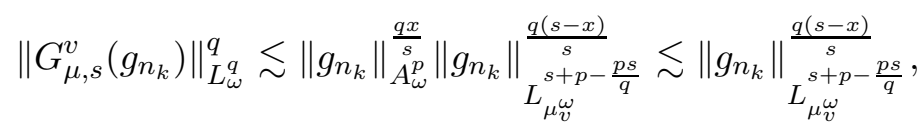

$$
\lim _{k \rightarrow \infty}\left\|G_{\mu, s}^{v}\left(g_{n_{k}}\right)\right\|_{L_{\omega}^{q}}=0 .
$$

Now, by using Lemma 7 and arguing as in the case $q>s$, we conclude that $\lim _{k \rightarrow \infty} \| G_{\mu, s}^{v}\left(f_{n_{k}}\right)-$ $G_{\mu, s}^{v}(f) \|_{L_{\omega}^{q}}=0$, that is, $G_{\mu, s}^{v}: A_{\omega}^{p} \rightarrow L_{\omega}^{q}$ is compact. 
Conversely, let $q<s$ and assume that $G_{\mu, s}^{v}: A_{\omega}^{p} \rightarrow L_{\omega}^{q}$ is bounded. Choose $\alpha>\beta>1$ such that $\frac{\beta}{\alpha}=\frac{q}{s}$. By arguing as in (3.1) and (3.2), we get

and

$$
\frac{\mu_{v}^{\omega}(S(a))}{\omega(S(a))^{s / p}} \lesssim\left\|G_{\mu, s}^{v}\left(f_{a, p}\right)\right\|_{L_{\omega}^{q}}^{\frac{q}{\beta}}\left\|G_{\mu, 1}^{v}\left(f_{a, p}^{s}(z) \chi_{S(a)}\right)\right\|_{L_{\omega}^{\frac{\beta^{\prime}}{\alpha^{\prime}}}}^{\frac{1}{\alpha^{\prime}}}
$$

$$
\left\|G_{\mu, 1}^{v}\left(f_{a, p}^{s}(z) \chi_{S(a)}\right)\right\|_{L_{\omega}^{\frac{\beta^{\prime}}{\alpha^{\prime}}}} \lesssim \frac{\mu_{v}^{\omega}(S(a))^{\frac{\alpha^{\prime}}{\beta^{\prime}}}}{\omega(S(a))^{s / p}}\left(\sup _{z \in \mathbb{D}} \frac{\mu_{v}^{\omega}(S(z) \cap S(a))}{\omega(S(z))}\right)^{1-\frac{\alpha^{\prime}}{\beta^{\prime}}}, \quad a \in \mathbb{D},
$$

respectively. These estimates yield

$$
\frac{\mu_{v}^{\omega}(S(a))}{\omega(S(a))^{s / p}} \lesssim\left\|G_{\mu, s}^{v}\left(f_{a, p}\right)\right\|_{L_{\omega}^{q}}^{\frac{q}{\beta}} \frac{\mu_{v}^{\omega}(S(a))^{\frac{1}{\beta^{\prime}}}}{\omega(S(a))^{\frac{s}{p \alpha^{\prime}}}}\left(\sup _{z \in \mathbb{D}} \frac{\mu_{v}^{\omega}(S(z) \cap S(a))}{\omega(S(z))}\right)^{\frac{1}{\alpha^{\prime}}-\frac{1}{\beta^{\prime}}}, \quad a \in \mathbb{D},
$$

and thus

$$
\frac{\mu_{v}^{\omega}(S(a))}{\omega(S(a))^{\frac{q}{p}}} \lesssim\left\|G_{\mu, s}^{v}\left(f_{a, p}\right)\right\|_{L_{\omega}^{q}}^{q}\left(\sup _{z \in \mathbb{D}} \frac{\mu_{v}^{\omega}(S(z) \cap S(a))}{\omega(S(z))}\right)^{\frac{\beta}{\alpha^{\prime}}-\frac{\beta}{\beta^{\prime}}}, \quad a \in \mathbb{D} .
$$

If $q=p$, we may use $\lim _{|a| \rightarrow 1^{-}}\left\|G_{\mu, s}^{v}\left(f_{a, p}\right)\right\|_{L_{\omega}^{p}}=0$ and Theorem 5 to deduce that the righthand side of (4.2) tends to zero as a approaches the boundary. Therefore $I_{d}: A_{\omega}^{p} \rightarrow L_{\mu_{v}^{\omega}}^{p}$ is compact by Theorem 3 (ii).

If $q>p$, by using (4.2) and arguing as in the corresponding part of the proof of Theorem 5 , we get

$$
\frac{\mu_{v}^{\omega}(S(a))}{\omega(S(a))^{1+\frac{s}{p}-\frac{s}{q}}} \leq\left\|G_{\mu, s}^{v}\left(f_{a, p}\right)\right\|_{L_{\omega}^{q}}^{q}\left(\sup _{b \in \mathbb{D}} \frac{\mu_{v}^{\omega}(S(b))}{\omega(S(b))^{1+\frac{s}{p}-\frac{s}{q}}}\right)^{1-\frac{q}{s}}, \quad a \in \mathbb{D} .
$$

from which arguments similar to those applied in the previous paragraph show that $I_{d}: A_{\omega}^{p} \rightarrow$ $L_{\mu_{v}^{\omega}}^{p+s-\frac{p s}{q}}$ is compact. This finishes the proof.

Theorem 9. Let $0<q<p<\infty$ and $0<s<\infty$ such that $1+\frac{s}{p}-\frac{s}{q}>0, \omega \in \widehat{\mathcal{D}}$ and let $\mu, v$ be positive Borel measures on $\mathbb{D}$ such that $\mu(\{z \in \mathbb{D}: v(T(z))=0\})=0=\mu(\{0\})$. Then the following conditions are equivalent:

(i) $G_{\mu, s}^{v}: A_{\omega}^{p} \rightarrow L_{\omega}^{q}$ is compact;

(ii) $G_{\mu, s}^{v}: A_{\omega}^{p} \rightarrow L_{\omega}^{q}$ is bounded;

(iii) $I_{d}: A_{\omega}^{p} \rightarrow L_{\mu_{v}^{\omega}}^{p\left(1+\frac{s}{p}-\frac{s}{q}\right)}$ is compact;

(iv) $I_{d}: A_{\omega}^{p} \rightarrow L_{\mu_{v}^{\omega}}^{p\left(1+\frac{s}{p}-\frac{s}{q}\right)}$ is bounded.

Proof. The conditions (ii)-(iv) are equivalent by Theorems 4(i) and 3)(iii). To complete the proof, it suffices to show that $G_{\mu, s}^{v}: A_{\omega}^{p} \rightarrow L_{\omega}^{q}$ is compact if $I_{d}: A_{\omega}^{p} \rightarrow L_{\mu_{v}^{\omega}}^{p\left(1+\frac{s}{p}-\frac{s}{q}\right)}$ is bounded. To see this, let $\left\{f_{n}\right\}$ be a bounded sequence in $A_{\omega}^{p}$, let $\left\{f_{n_{k}}\right\}$ be a subsequence that converges uniformly on compact subsets of $\mathbb{D}$ to $f \in A_{\omega}^{p}$. Write $g_{k}=f_{n_{k}}-f$ as before. By using Theorem 3 and the last part of the proof of Theorem 3(iii), we deduce

$$
\lim _{R \rightarrow 1^{-}} \int_{\mathbb{D}}\left(\int_{\Gamma(\zeta) \backslash \overline{D(0, R)}} \frac{d \mu(z)}{\omega(T(z))}\right)^{\frac{p q}{s(p-q)}} \omega(\zeta) d A(\zeta)=0 .
$$

Therefore, for a fixed $\varepsilon>0$, there exists $R_{0} \in(0,1)$ such that

$$
\int_{\mathbb{D}}\left(\int_{\Gamma(\zeta) \backslash \overline{D\left(0, R_{0}\right)}} \frac{d \mu(z)}{\omega(T(z))}\right)^{\frac{p q}{s(p-q)}} \omega(\zeta) d A(\zeta)<\varepsilon^{\frac{p}{p-q}} .
$$


Choose $k_{0} \in \mathbb{N}$ such that $\left|g_{k}(z)\right|<\varepsilon^{1 / q}$ for all $k \geq k_{0}$ and $z \in \overline{D\left(0, R_{0}\right)}$. Then Hölder's inequality and [12, Lemma 4.4] give

$$
\begin{aligned}
\left\|G_{\mu, s}^{v}\left(g_{k}\right)\right\|_{L_{\omega}^{q}}^{q} \lesssim & \int_{\mathbb{D}}\left(\int_{\Gamma(z) \cap \overline{D\left(0, R_{0}\right)}}\left|g_{k}(\zeta)\right|^{s} \frac{d \mu(\zeta)}{v(T(\zeta))}\right)^{\frac{q}{s}} \omega(z) d A(z) \\
& +\int_{\mathbb{D}}\left(\int_{\Gamma(z) \backslash \overline{D\left(0, R_{0}\right)}}\left|g_{k}(\zeta)\right|^{s} \frac{d \mu(\zeta)}{v(T(\zeta))}\right)^{\frac{q}{s}} \omega(z) d A(z) \\
\lesssim & +\int_{\mathbb{D}} N\left(g_{k}\right)^{q}(z)\left(\int_{\Gamma(z) \backslash \overline{D\left(0, R_{0}\right)}} \frac{d \mu(\zeta)}{v(T(\zeta))}\right)^{\frac{q}{s}} \omega(z) d A(z) \\
\leq & +\left\|N\left(g_{k}\right)\right\|_{L_{\omega}^{p}}^{q}\left(\int_{\mathbb{D}}\left(\int_{\Gamma(z) \backslash \overline{D\left(0, R_{0}\right)}} \frac{d \mu(\zeta)}{v(T(\zeta))}\right)^{\frac{p q}{(p-q) s}} \omega(z) d A(z)\right)^{\frac{p-q}{p}} \lesssim \varepsilon
\end{aligned}
$$

and consequently, $\lim _{k \rightarrow \infty}\left\|G_{\mu, s}^{v}\left(g_{k}\right)\right\|_{L_{\omega}^{q}}^{q}=0$. Finally, by using Lemma 7 and arguing as in the proof of Theorem 8 , we deduce $\lim _{k \rightarrow \infty}\left\|G_{\mu, s}^{v}\left(f_{k}\right)-G_{\mu, s}^{v}(f)\right\|_{L_{\omega}^{q}}^{q}=0$, that is, $G_{\mu, s}^{v}: A_{\omega}^{p} \rightarrow L_{\omega}^{q}$ is compact.

\section{Applications AND FURThER COMMENTS}

5.1. Area operators in Hardy spaces. For $0<s<\infty$, define

$$
G_{\mu, s}(f)(z)=\left(\int_{\Gamma(z)}|f(\zeta)|^{s} \frac{d \mu(\zeta)}{1-|\zeta|}\right)^{\frac{1}{s}}, \quad z \in \mathbb{T} .
$$

The method of proof of Theorem 4, combined with the results in [9, Section 7] and [10], can be used to obtain the following result. The details of the proof does not reveal anything new, and are therefore omitted. Here $L^{q}(\mathbb{T})$ refers to the classical $L^{q}$-space on $\mathbb{T}$.

Theorem 10. Let $0<p, q<\infty$ such that $1+\frac{s}{p}-\frac{s}{q}>0$, and let $\mu$ be a positive Borel measure on $\mathbb{D}$ such that $\mu(\{0\})=0$. Then $G_{\mu, s}: H^{p} \rightarrow L^{q}(\mathbb{T})$ is bounded (resp. compact) if and only if $I_{d}: H^{p} \rightarrow L_{\mu}^{p+s-\frac{p s}{q}}$ is bounded (resp. compact). Moreover,

$$
\left\|G_{\mu, s}\right\|_{H^{p} \rightarrow L^{q}(\mathbb{T})}^{s} \asymp\|I d\|_{H^{p} \rightarrow L_{\mu}^{p+s-\frac{p s}{q}}}^{p+s-\frac{p s}{q}} \asymp \sup _{a \in \mathbb{D}} \frac{\mu(S(a))}{(1-|a|)^{1+\frac{s}{p}-\frac{s}{q}}}, \quad p \leq q,
$$

and

$$
\left\|G_{\mu, s}\right\|_{H^{p} \rightarrow L^{q}(\mathbb{T})}^{s} \asymp\|I d\|_{H^{p} \rightarrow L_{\mu}^{p+s-\frac{p s}{q}}}^{p+s-\frac{p s}{q}} \asymp\left\|B_{\mu}\right\|_{L^{\frac{q p}{s(p-q)}}}, \quad q<p
$$

where

$$
B_{\mu}(\zeta)=\int_{\Gamma(\zeta)} \frac{d \mu(z)}{1-|z|}, \quad \zeta \in \mathbb{T}
$$

In particular, this result proves the conjecture in [8, p. 365] in the case $1+\frac{1}{p}-\frac{1}{q}>0$.

5.2. Integral operator $T_{g}$ on Bergman and Hardy spaces. Each $g \in \mathcal{H}(\mathbb{D})$ induces the integral operator

$$
T_{g}(f)(z)=\int_{0}^{z} g^{\prime}(\zeta) f(\zeta) d \zeta, \quad z \in \mathbb{D}
$$

acting on $\mathcal{H}(\mathbb{D})$. This type of integral operators have been extensively studied during the last decades and have interesting connections with other areas of mathematical analysis, see [12, 11] and the references therein. In particular, the symbols $g$ for which $T_{g}$ is bounded or compact from $A_{\omega}^{p}$ to $A_{\omega}^{q}$ can be described in terms of the following spaces of analytic functions when $q \geq p$. 
We say that $g \in \mathcal{H}(\mathbb{D})$ belongs to $\mathcal{C}^{q, p}\left(\omega^{\star}\right), 0<p, q<\infty$, if the measure $\left|g^{\prime}(z)\right|^{2} \omega^{\star}(z) d A(z)$ is a $q$-Carleson measure for $A_{\omega}^{p}$. Moreover, $g \in \mathcal{C}_{0}^{q, p}\left(\omega^{\star}\right)$ if the identity operator $I_{d}: A_{\omega}^{p} \rightarrow$ $L^{q}\left(\left|g^{\prime}\right|^{2} \omega^{\star} d A\right)$ is compact. If $q \geq p$ and $\omega \in \widehat{\mathcal{D}}$, then Theorem 3 shows that these spaces only depend on the quotient $\frac{q}{p}$. Consequently, for $q \geq p$ and $\omega \in \widehat{\mathcal{D}}$, we simply write $\mathcal{C}^{q / p}\left(\omega^{\star}\right)$ instead of $\mathcal{C}^{q, p}\left(\omega^{\star}\right)$. Thus, if $\alpha \geq 1$ and $\omega \in \widehat{\mathcal{D}}$, then $\mathcal{C}^{\alpha}\left(\omega^{\star}\right)$ consists of those $g \in \mathcal{H}(\mathbb{D})$ such that

$$
\|g\|_{\mathcal{C}^{\alpha}\left(\omega^{\star}\right)}^{2}=|g(0)|^{2}+\sup _{I \subset \mathbb{T}} \frac{\int_{S(I)}\left|g^{\prime}(z)\right|^{2} \omega^{\star}(z) d A(z)}{(\omega(S(I)))^{\alpha}}<\infty .
$$

An analogue of this identity is valid for the little space $\mathcal{C}_{0}^{\alpha}\left(\omega^{\star}\right)$. We refer to [12, Chapter 5] for further information about these spaces.

Theorem 11. Let $0<p, q<\infty$ such that $q>\frac{2 p}{2+p}$ and $\omega \in \widehat{\mathcal{D}}$. Let $g \in \mathcal{H}(\mathbb{D})$ and denote $d \mu_{g}(z)=\left|g^{\prime}(z)\right|^{2} \omega^{\star}(z) d A(z)$. Then $T_{g}: A_{\omega}^{p} \rightarrow A_{\omega}^{q}$ is bounded (resp. compact) if and only if $I_{d}: A_{\omega}^{p} \rightarrow L_{\mu_{g}}^{p+2-\frac{2 p}{q}}$ is bounded (resp. compact).

Proof. By [12, Theorem 4.2], $T_{g}: A_{\omega}^{p} \rightarrow L_{\omega}^{q}$ is bounded (resp. compact) if and only if $G_{\mu_{g}, 2}^{\omega}: A_{\omega}^{p} \rightarrow L_{\omega}^{q}$ is bounded (resp. compact), and $\left\|T_{g}\right\|_{A_{\omega}^{p} \rightarrow L_{\omega}^{q}} \asymp\left\|G_{\mu_{g}, 2}^{\omega}\right\|_{A_{\omega}^{p} \rightarrow L_{\omega}^{q}}$. Theorem 4 implies

and this finishes the proof.

$$
\left\|G_{\mu_{g}, 2}^{\omega}\right\|_{A_{\omega}^{p} \rightarrow L_{\omega}^{q}}^{2} \asymp\left\|I_{d}\right\|_{A_{\omega}^{p} \rightarrow L_{\mu_{g}}^{p+2-\frac{2 p}{q}}}^{p+2-\frac{2 p}{q}},
$$

It is worth mentioning that Theorem 3 yields

$$
\left\|T_{g}\right\|_{A_{\omega}^{p} \rightarrow L_{\omega}^{q}}^{2} \asymp \sup _{a \in \mathbb{D}} \frac{\mu_{g}(S(a))}{\omega(S(a))^{2\left(\frac{1}{p}-\frac{1}{q}\right)+1}}, \quad q \geq p
$$

Thus $T_{g}: A_{\omega}^{p} \rightarrow L_{\omega}^{q}$ is bounded if and only if $g \in \mathcal{C}^{2\left(\frac{1}{p}-\frac{1}{q}\right)+1}\left(\omega^{\star}\right)$. If $p>q$, Theorem 3 also gives

$$
\left\|T_{g}\right\|_{A_{\omega}^{p} \rightarrow L_{\omega}^{q}}^{2} \asymp \int_{\mathbb{D}}\left(\int_{\Gamma(\zeta)}\left|g^{\prime}(z)\right|^{2} d A(z)\right)^{\frac{q p}{2(p-q)}} \omega(\zeta) d A(\zeta),
$$

and thus $T_{g}: A_{\omega}^{p} \rightarrow L_{\omega}^{q}$ is bounded if and only if $g \in A_{\omega}^{\frac{q p}{p-q}}$ by [12, Theorem 4.2].

Consequently, whenever $q>\frac{2 p}{2+p}$ Theorem 11 improves [12, Theorem 4.1] because the hypothesis on $\omega$ are stronger in the original result. In particular, if $q<p$, the weight $\omega$ is assumed to be continuous and strictly positive with the local regularity

$$
\omega(t) \asymp \omega(r), \quad 1-t \asymp 1-r .
$$

This hypothesis allows one to use the strong factorization $A_{\omega}^{p}=A_{\omega}^{p_{1}} \cdot A_{\omega}^{p_{2}}, p^{-1}=p_{1}^{-1}+p_{2}^{-1}$, [12, Theorem 3.1], which is a principal ingredient in the proof of [12, Theorem 4.1].

However, the defect of Theorem 11 is the extra hypothesis $q>\frac{2 p}{2+p}$ which is a restriction only in the case $p>q$. This condition is inherited from Theorem 4 and appears there because Carleson measures are finite measures. This is not true in general for $\mu_{g}$ when $g \in A_{\omega}^{\frac{q p}{p-q}}$ and $q<\frac{2 p}{2+p}$. The case of compact operators can be analyzed in the same way.

If $q>\frac{2 p}{2+p}$ one may characterize bounded and compact operators $T_{g}: H^{p} \rightarrow H^{q}$ by using Section 5.1. In order to avoid unnecessary repetition, we omit the details.

\section{REFERENCES}

[1] C. Cascante and J. Ortega, Imbedding potentials in tent spaces, J. Funct. Anal. 198 (2003), no. 1, $106-141$.

[2] W. S. Cohn, Generalized area operators on Hardy spaces, J. Math. Anal. Appl. 216 (1997), no. 1, $112-121$.

[3] W. S. Cohn and I. E. Verbitsky, Factorization of tent spaces and Hankel operators, J. Funct. Anal. 175 (2000), no. 2, 308-329. 
[4] R. R. Coifman, Y. Meyer and E. M. Stein, Some new functions spaces and their applications to Harmonic Analysis, J. Funct. Anal. 62 (1985), no. 3, 304-335.

[5] P. Duren, Theory of $H^{p}$ Spaces, Academic Press, New York-London 2000.

[6] C. Fefferman and E. M. Stein, $H^{p}$ spaces of several variables, Acta Math. 129 (1972), no. 3-4, $137-193$.

[7] J. Garnett, Bounded analytic functions, Academic Press, New York, 1981.

[8] M. Gong, Z. Lou and Z. Wu, Area operators from $H^{p}$ spaces to $L^{q}$ spaces, Sci. China Math. 53 (2010), no. $2,357-366$.

[9] D. H. Luecking, Embedding derivatives of Hardy spaces into Lebesgue spaces, Proc. London Math. Soc. 63 (1991), no. 3, 595-619.

[10] J.A. Peláez, Compact embedding derivatives of Hardy spaces into Lebesgue spaces, to appear in Proc. Amer. Math. Soc. http://arxiv.org/abs/1502.05527

[11] J.A. Peláez, Small weighted Bergman spaces, Proceedings of the Summer School "Complex and harmonic analysis and related topics" Mekrijärvi, June (2014).

[12] J. A. Peláez and J. Rättyä, Weighted Bergman spaces induced by rapidly increasing weights, Mem. Amer. Math. Soc. 227 (2014), no. 1066.

[13] J.A. Peláez and J.Rättyä, Embedding theorems for Bergman spaces via harmonic analysis, Math. Ann. (to appear), DOI 10.1007/s00208-014-1108-5

[14] J. A. Peláez and J. Rättyä, On the boundedness of Bergman projection, to appear, VI CIDAMA 2014, http://arxiv.org/abs/1501.03957

Departamento de Análisis Matemático, Universidad de Málaga, Campus de Teatinos, 29071 MÁlagA, SPAIN

E-mail address: japelaez@uma.es

University of Eastern Finland, P.O. Box 111, 80101 Joensuu, Finland

E-mail address: jouni.rattya@uef.fi

University of Eastern Finland, P.O. Box 111, 80101 Joensuu, Finland; Departamento de Análisis Matemático, Universidad de Málaga, Campus de Teatinos, 29071 Málaga, Spain

E-mail address: kiansierra@hotmail.com 